\title{
Alpha-1 acid glycoprotein reduction differentiates recovery from remission in feline infectious peritonitis treatment
}

\author{
Diane D. Addie ( $\sim$ draddie@catvirus.com ) \\ catvirus.com \\ Carla Silveira \\ Medivet Canonbury \\ Charlotte Aston \\ Melton Vets \\ Pauline Brauckmann \\ Ãstersundsdjursjukhuset \\ Johanna Covell-Ritchie \\ Chris Felstead \\ Kynoch and Partners \\ Mark Fosbery \\ Newnham Vets \\ Caryn Gibbins \\ Zasman Vet \\ Kristina Macaulay \\ Belle Ayr Cats \\ James McMurrough \\ Vets Now \\ Ed Pattison \\ City Vets \\ Elise Robertson \\ Endsocopy Vet Referrals
}

\section{Research Article}

Keywords: feline coronavirus, feline infectious peritonitis, FIP, treatment, alpha- 1 acid glycoprotein, AGP, acute phase protein, interferon omega, antivirals, meloxicam.

Posted Date: January 19th, 2022

DOl: https://doi.org/10.21203/rs.3.rs-1145695/v1

License: (c) (i) This work is licensed under a Creative Commons Attribution 4.0 International License. Read Full License 


\section{Abstract}

Feline infectious peritonitis (FIP) is a systemic immune-mediated inflammatory perivasculitis which occurs in a minority of cats infected with feline coronavirus (FCoV). A variety of therapies have been employed to treat this condition, which previously was usually fatal, but no parameters for differentiating FIP recovery from remission have been defined to enable clinicians to decide when it is safe to discontinue treatment. This observational study shows that a consistent reduction of the acute phase protein alpha-1 acid glycoprotein (AGP) to within normal limits (WNL i.e. $500 \mu \mathrm{g} / \mathrm{ml}$ or below), as opposed to duration of survival, distinguishes recovery from remission. AGP consistently reduced to WNL in 26 recovered cats but remained elevated in 16 cats in remission, dipping to normal once in two of the latter. Anaemia was present in 58\% (23/40) of the cats, and resolved more quickly than AGP in six recovered cats. Lymphopenia was observed in $43 \%(16 / 37)$ of the cats and reversed in nine recovered cats, but none of the remission group. Hyperglobulinaemia was slower than AGP to return to WNL in the recovered cats. FCoV antibody titre was high in all 42 cats at the outset and decreased significantly in 7 recovered cats, but too slowly to be a useful parameter to determine discontinuation of antiviral treatments. Conclusion: a sustained return to normal levels of AGP was the most rapid and consistent indicator for differentiating recovery from remission following treatment for FIP. This study provides a useful model for differentiating acute from chronic coronavirus disease using acute phase protein monitoring.

\section{Introduction}

Feline coronavirus (FCoV) is a positive strand RNA virus belonging to the order Nidovirales, genus Coronavirus, and family Coronaviridae, subfamily alphacoronavirus. The FCoV species is further divided into two types: I and II, the first type being wholly feline, type II FCoV arising from recombination events with canine coronavirus ${ }^{1,2}$.

Feline infectious peritonitis (FIP) is an immune-mediated perivascular pyogranulomatous ${ }^{3}$. disease which affects $5-10 \%$ of cats infected with FCoV ${ }^{4}$.. FIP can present acutely, with effusions in one or more body cavities, or as a disease of chronic inflammation, with cachexia and variable organ damage depending on the sites of pyogranuloma formation.

The initial target for FCoV is the epithelial cells of the small intestine; from there macrophages engulf the virus and transport it to the mesenteric lymph nodes $(M L N)$ and a brief systemic phase of infection follows when the target cell is the monocyte.

In FIP, coronavirus-infected monocytes/macrophages release matrix metalloproteinase-9 (MMP-9) ${ }^{3}$. MMP-9 allows the extravasation of FCoV-infected monocytes, which differentiate into macrophages. FCoV-infected macrophages release a storm of cytokines and chemokines including tumour necrosis factor-alpha (TNF-a) ${ }^{3,5,6}$ and interleukin-6 (IL-6) ${ }^{7}$.

TNF-a is a major contributor to the inflammatory response and pathogenesis of FIP. FCoV-infected cells release a substance which causes apoptosis in nearby lymphocytes ${ }^{8}$ : the mystery substance is probably TNF- $a^{5}$ : around $50 \%$ of cats with FIP are lymphopenic $9,10,11$.

IL-6 stimulates hepatocytes to release acute phase proteins ${ }^{7}$ such as alpha-1 acid glycoprotein (AGP) and serum amyloid A. AGP was first reported to be elevated in FIP cases in $1997^{12}$. Raised AGP has been shown to be superior to histopathology of biopsy in differentiating FIP from similarly-presenting cases ${ }^{13}$. Monitoring AGP is an accurate predictor of survival in humans with sepsis ${ }^{14}$.

Many treatments, ranging from prednisolone, through feline interferon omega ( $\mathrm{rFelFN}$ omega) ${ }^{15}$, polyprenyl immunostimulant ${ }^{16}$, meloxicam ${ }^{17}$, to, most recently, specific antivirals ${ }^{18,19}$ have been used. Those treatments involved in the present study are detailed in Tables 1 and 2 .

Page $2 / 31$ 
Table 1

How FIP was diagnosed, survival time and treatment details of 26 cats who recovered

\begin{tabular}{|c|c|c|c|c|c|c|c|}
\hline & Cat & $\begin{array}{l}\text { FIP } \\
\text { presentation }\end{array}$ & $\begin{array}{l}\text { How FIP } \\
\text { diagnosed }\end{array}$ & $\begin{array}{l}\text { Survival } \\
\text { in years } \\
\text { or months }\end{array}$ & $\begin{array}{l}\text { Time to } \\
\text { normal } \\
\text { AGP }\end{array}$ & Treatments & Prednisolone \\
\hline 1 & Basil 1 & $\begin{array}{l}\text { Non- } \\
\text { effusive } \\
\text { (icterus) }\end{array}$ & $\begin{array}{l}\text { mRNA RT-PCR } \\
\text { positive pbmc } \\
\text { thrice }\end{array}$ & $11 y$ & $<22 d$ & $\begin{array}{l}5 \times 10^{4} \text { units of } \\
\text { rFelFN- } \omega \text { per os } \\
\text { q24h for } 13 \mathrm{~m} \\
\text { until FCoV } \\
\text { antibody titre } \\
\text { reduced from } \\
>1280 \text { to }<1: 10 \text {. } \\
\\
\text { He died of chronic } \\
\text { kidney disease } \\
\text { aged } 15 y \text {. }\end{array}$ & $\begin{array}{l}2 \mathrm{mg} / \mathrm{kg} \mathrm{q} 24 \mathrm{~h} \text { for } \\
7 \mathrm{~d} \text { then } 1 \mathrm{mg} / \mathrm{kg} \\
\text { for } 7 \mathrm{~d}\end{array}$ \\
\hline 2 & Boris & $\begin{array}{l}\text { Non- } \\
\text { effusive but } \\
\text { initially } \\
\text { effusive FIP } \\
\text { suspected }\end{array}$ & $\begin{array}{l}\text { MLN FNA RT- } \\
\text { qPCR positive } \\
\mathrm{C}_{T} 33.7 . \\
\text { Ascites RT- } \\
\text { qPCR } \\
\text { negative. }\end{array}$ & $>2.2 y$ & $<3 \mathrm{~m}$ & $\begin{array}{l}\text { 1MU units rFelFN- } \\
\omega \text { per os q24h. PI } \\
3 \mathrm{mg} / \mathrm{kg} \text { per os } \\
\text { q72h. Weekly } \\
\text { cobalamin } \\
\text { injections. } \\
\text { Effusion was } \\
\text { negative on RT- } \\
\text { PCR and was } \\
\text { found to be due } \\
\text { to } \\
\text { cardiomyopathy. }\end{array}$ & No \\
\hline 3 & Mars & $\begin{array}{l}\text { Non- } \\
\text { effusive }\end{array}$ & $\begin{array}{l}\text { MLN FNA RT- } \\
\text { qPCR } C_{T} 30\end{array}$ & $>5.5 y$ & $<6 \mathrm{~m}$ & $\begin{array}{l}\mathrm{PI} 3 \mathrm{mg} / \mathrm{kg} \text { twice } \\
\text { per week. }\end{array}$ & No \\
\hline 4 & Chester & $\begin{array}{l}\text { Effusive } \\
\text { (pleural } \\
\text { effusion) }\end{array}$ & $\begin{array}{l}\mathrm{C}_{T} 34 \mathrm{RT}-\mathrm{qPCR} \\
\text { on pleural } \\
\text { effusion }\end{array}$ & $>3.1 y$ & $<8 m$ & $\begin{array}{l}1 \mathrm{MU} / \mathrm{kg} \text { rFelFN- } \omega \\
\text { s/c q48 h reduced } \\
\text { to q4d then } 1 \mathrm{x} \\
10^{5} \text { units per os } \\
\text { q24h for } 28 \mathrm{~m} . \\
\text { Meloxicam. SAMe } \\
\text { (Denamarin) } \\
\text { Cobalamin } \\
\text { (Cobalaplex). } \\
\text { Tramadol } \\
\text { hydrochloride } \\
\text { (Bova } \\
\text { Compounding, } \\
\text { UK) } 10 \text { mg /cat } \\
\text { q24h per os. (Hugo } \\
\text { and Heading 2015) } \\
\text { Liquorice tea was } \\
\text { attempted but cat } \\
\text { did not like it. }\end{array}$ & $\begin{array}{l}1 \mathrm{mg} / \mathrm{kg} \mathrm{q} 12 \mathrm{~h} \text { for } \\
2 \mathrm{w} \text {, weaned off } \\
\text { for another } 3 \mathrm{w} \\
\text { and replaced by } \\
\text { meloxicam }\end{array}$ \\
\hline 5 & Amy & $\begin{array}{l}\text { Non- } \\
\text { effusive }\end{array}$ & FIP profile & $>1.3 y$ & $<4 \mathrm{~m}$ & $\begin{array}{l}1 \mathrm{MU} \text { units rFelFN- } \\
\omega \mathrm{s} / \mathrm{c} \mathrm{q} 48 \mathrm{~h} .\end{array}$ & Sliding doses \\
\hline 6 & Brook & $\begin{array}{l}\text { Non- } \\
\text { effusive }\end{array}$ & FIP profile & $>1.1 y$ & $<41 \mathrm{~d}$ & Unknown. & Unknown \\
\hline
\end{tabular}




\begin{tabular}{|c|c|c|c|c|c|c|c|}
\hline & Cat & $\begin{array}{l}\text { FIP } \\
\text { presentation }\end{array}$ & $\begin{array}{l}\text { How FIP } \\
\text { diagnosed }\end{array}$ & $\begin{array}{l}\text { Survival } \\
\text { in years } \\
\text { or months }\end{array}$ & $\begin{array}{l}\text { Time to } \\
\text { normal } \\
\text { AGP }\end{array}$ & Treatments & Prednisolone \\
\hline 7 & Basil 2 & $\begin{array}{l}\text { Effusive } \\
\text { (ascites) }\end{array}$ & $\begin{array}{l}\text { RT-qPCR } \\
\text { positive on } \\
\text { ascites }\end{array}$ & $>2.5 y$ & $<10 w$ & $\begin{array}{l}1 \mathrm{MU} / \mathrm{kg} \text { rFelFN- } \omega \\
\text { s/c q24h for } 36 \mathrm{~d}, \\
\text { reducing to every } \\
3 \mathrm{~d} \text {, followed by } 1 \\
\times 10^{5} \text { units of } \\
\text { rFelFN- } \omega \text { per os } \\
\text { q24h for } 2 \mathrm{y} \text {. Pl at } \\
3 \mathrm{mg} / \mathrm{kg} \text { q48h } \\
\text { during } 10 \mathrm{~d} \text {. } \\
\text { Mirtazapine. } \\
\text { Ursodeoxychloic } \\
\text { acid (Ursodiol, } \\
\text { USA). Cobalamin } \\
\text { injections weekly. } \\
\text { Itraconazole } \\
\text { 10mg/kg from } \\
\text { days } 4-87 \text {. One } \\
\text { Darbepoetin } \\
\text { injection. GC-376 } \\
\text { s/c Days } 17-100 . \\
\text { Doxycycline } \\
10 m g / k g \text { bid per } \\
\text { os from d.32 for } \\
\text { 30d (to treat } \\
\text { haemotropic } \\
\text { mycoplasmosis). }\end{array}$ & $\begin{array}{l}\text { Only for 3d: } \\
\text { meloxicam used } \\
\text { in preference } \\
\text { from Day } 4\end{array}$ \\
\hline 8 & Kitten 2 & $\begin{array}{l}\text { Effusive } \\
\text { (ascites) } \\
\text { then non- } \\
\text { effusive }\end{array}$ & FIP Profile & $\begin{array}{l}>2 y \text { post } \\
\text { FIP } \\
\text { diagnosis, } \\
>1.8 \mathrm{y} \\
\text { post } \\
\text { relapse }\end{array}$ & $<6 \mathrm{~m}$ & $\begin{array}{l}57 \mathrm{~d} \text { of Mutian X } \\
\text { at } 80 \mathrm{mg} / \mathrm{kg} \text {. } \\
\text { Following her } \\
\text { neurological } \\
\text { relapse, she was } \\
\text { re-treated with } \\
160 \mathrm{mg} / \mathrm{kg} \text { for } \\
2 \mathrm{~m} .1 \times 10^{5} \text { units } \\
\text { of rFelFN- } \omega \text { per } \\
\text { os q24h. }\end{array}$ & No \\
\hline 9 & Skywise & $\begin{array}{l}\text { Non- } \\
\text { effusive }\end{array}$ & $\begin{array}{l}\text { MLN FNA RT- } \\
\text { qPCR positive }\end{array}$ & $>1.5 y$ & $<35 d$ & $\begin{array}{l}\text { 50d Mutian X } \\
\text { starting } \\
160 \mathrm{mg} / \mathrm{kg} \text { q24h } \\
\text { per os in divided } \\
\text { doses, reduced to } \\
120 \mathrm{mg} / \mathrm{kg} \text { on } \\
\text { Day } 25 ; \text { followed } \\
\text { by } 1 \times 10^{5} \text { units } \\
\text { of rFelFN- } \omega \text { per } \\
\text { os q24h. } \\
\text { Cobalamin } \\
\text { (Cobalaplex). }\end{array}$ & $\begin{array}{l}6 \mathrm{~d} \text { per os then in } \\
\text { eye drops for } 14 \mathrm{~d}\end{array}$ \\
\hline 10 & Betsy & $\begin{array}{l}\text { Effusive } \\
\text { (ascites) }\end{array}$ & $\begin{array}{l}\text { RT-qPCR } \\
\text { positive on } \\
\text { ascites } C_{T} \\
25.6\end{array}$ & $>1.5 y$ & $<39 d$ & $\begin{array}{l}38 \mathrm{~d} \text { Mutian } \mathrm{X} \\
80 \mathrm{mg} / \mathrm{kg} \text { per os } \\
\text { for } 31 \mathrm{~d} \text { and } \\
160 \mathrm{mg} / \mathrm{kg} \text { for } 7 \mathrm{~d} \\
\text { followed by } 1 \mathrm{x} \\
10^{5} \text { units of } \\
\text { rFelFN- } \omega \text { per os } \\
\text { q24h. } \\
\text { Doxycycline. }\end{array}$ & $\begin{array}{l}5 \mathrm{mg} / \mathrm{cat} \mathrm{q} 24 \mathrm{~h} \\
\text { for } 10 \mathrm{~d} \text { replaced } \\
\text { by meloxicam }\end{array}$ \\
\hline
\end{tabular}




\begin{tabular}{|c|c|c|c|c|c|c|c|}
\hline & Cat & $\begin{array}{l}\text { FIP } \\
\text { presentation }\end{array}$ & $\begin{array}{l}\text { How FIP } \\
\text { diagnosed }\end{array}$ & $\begin{array}{l}\text { Survival } \\
\text { in years } \\
\text { or months }\end{array}$ & $\begin{array}{l}\text { Time to } \\
\text { normal } \\
\text { AGP }\end{array}$ & Treatments & Prednisolone \\
\hline 11 & Dante & Colonic FIP & $\begin{array}{l}\text { Histopath of } \\
\text { biopsy }\end{array}$ & $>7 \mathrm{~m}$ & $<28 d$ & $\begin{array}{l}\text { 84d of Mutian X } \\
\text { 80mg/kg per os. } \\
\text { Protexin pro-kolin } \\
\text { enterogenic } \\
\text { probiotics } 2 \mathrm{ml} \text { per } \\
\text { os q12h. } \\
\text { Cobalamin } \\
\text { (Cobalaplex). }\end{array}$ & $\begin{array}{l}\text { No: gabapentin } \\
25 \mathrm{mg} \text { q8h }\end{array}$ \\
\hline 12 & Elmo & $\begin{array}{l}\text { Non- } \\
\text { effusive }\end{array}$ & $\begin{array}{l}\text { Biopsy } \\
\text { histopath } \\
\text { reported } \\
\text { pyogranuloma }\end{array}$ & $>8 m$ & $<31 d$ & $\begin{array}{l}54 \mathrm{~d} \text { Mutian } \mathrm{X} \\
(160 \mathrm{mg} / \mathrm{kg} \text { for } \\
37 \mathrm{~d} \text {, then } 80 \\
\mathrm{mg} / \mathrm{kg}) \text { followed } \\
\text { by } 1 \times 10^{5} \text { units } \\
\text { of rFelFN- } \omega \text { per } \\
\text { os q24h. }\end{array}$ & $\begin{array}{l}\text { One injection and } \\
\text { one } 5 \mathrm{mg} \text { pill } \\
\text { given once only }\end{array}$ \\
\hline 13 & Lyra & $\begin{array}{l}\text { Effusive } \\
\text { (ascites) }\end{array}$ & $\begin{array}{l}\text { RT-qPCR low } \\
\text { positive on } \\
\text { ascites: } C_{T} 33\end{array}$ & $>8 m$ & $<20 d$ & $\begin{array}{l}47 \mathrm{~d} \text { Mutian X } \\
80 \mathrm{mg} / \mathrm{kg} \text { q24h } \\
\text { (divided doses) } \\
\text { per os followed } \\
\text { by } 1 \times 10^{5} \text { units } \\
\text { of rFelFN-w per } \\
\text { os q24h for } 5 \mathrm{~m} \text {. }\end{array}$ & $\begin{array}{l}\text { No: meloxicam } \\
\text { injections }\end{array}$ \\
\hline 14 & Molly & $\begin{array}{l}\text { Non- } \\
\text { effusive }\end{array}$ & $\begin{array}{l}\text { Profile, RT-PCR } \\
\text { positive } \\
\text { faeces over } 24 \\
\mathrm{~m}\end{array}$ & $>3.3 y$ & $<16 \mathrm{~m}$ & $\begin{array}{l}1 \times 10^{5} \text { units of } \\
\text { rFelFN- } \omega \text { per os } \\
\text { q24h, gabapentin, } \\
\text { cefovecin } \\
\text { (Convenia, Zoetis } \\
\text { UK), Protexin } \\
\text { Synbiotic D-C } \\
\text { probiotics. }\end{array}$ & $\begin{array}{l}\text { Sliding doses } \\
\text { (unspecified)then } \\
\text { meloxicam }\end{array}$ \\
\hline 15 & Bea & $\begin{array}{l}\text { Effusive } \\
\text { (ascites) }\end{array}$ & $\begin{array}{l}\text { Pos RT-PCR } \\
\text { on ascites }\end{array}$ & $\begin{array}{l}13 \mathrm{~m}+ \\
\text { (died of } \\
\text { cancer, } \\
\text { aged 8y) }\end{array}$ & $<69 d$ & $\begin{array}{l}\text { 94d of Mutian X: } \\
40 \mathrm{mg} / \mathrm{kg} \text { (i.e. half } \\
\text { dose) for } 4 \mathrm{~d} \text {, then } \\
80 \mathrm{mg} / \mathrm{kg} \text { for } 90 \mathrm{~d} \text {, } \\
\text { except for one } \\
\text { week of double } \\
\text { dose, followed by } \\
1 \times 10^{5} \text { units of } \\
\text { rFelFN- } \omega \text { per os } \\
\text { q24h for } 6 \mathrm{~m} . \\
\text { Robenacoxib } \\
\text { (Onsior, Elanco, } \\
\text { UK) given once } \\
\text { only. }\end{array}$ & No \\
\hline 16 & Buddie & $\begin{array}{l}\text { Effusive } \\
\text { (ascites) }\end{array}$ & $\begin{array}{l}\text { Biopsy and } \\
\text { positive RT- } \\
\text { PCR ascites: } \\
\mathrm{C}_{T} 33\end{array}$ & $>9 m$ & $<51 d$ & $\begin{array}{l}69 \mathrm{~d} \text { of Mutian } \mathrm{X} \\
80 \mathrm{mg} / \mathrm{kg} \text { with } \\
160 \mathrm{mg} / \mathrm{kg} \text { in the } \\
3 \mathrm{rd} \text { w of } \\
\text { treatment; } \\
\text { followed by } 1 \mathrm{x} \\
10^{5} \text { units of } \\
\text { rFelFN-w per os } \\
\text { q24h for } 6 \mathrm{~m} \text {. } \\
\text { Cobalamin } \\
\text { (Cobalaplex); } \\
\text { SAMe } \\
\text { (Denamarin). }\end{array}$ & $\begin{array}{l}\text { One } \\
\text { dexamethasone } \\
\text { injection only }\end{array}$ \\
\hline
\end{tabular}




\begin{tabular}{|c|c|c|c|c|c|c|c|}
\hline & Cat & $\begin{array}{l}\text { FIP } \\
\text { presentation }\end{array}$ & $\begin{array}{l}\text { How FIP } \\
\text { diagnosed }\end{array}$ & $\begin{array}{l}\text { Survival } \\
\text { in years } \\
\text { or months }\end{array}$ & $\begin{array}{l}\text { Time to } \\
\text { normal } \\
\text { AGP }\end{array}$ & Treatments & Prednisolone \\
\hline 17 & Nelson & $\begin{array}{l}\text { Non- } \\
\text { effusive } \\
\text { becoming } \\
\text { effusive }\end{array}$ & $\begin{array}{l}\text { IHC of MLN } \\
\text { biopsy }\end{array}$ & $>6 \mathrm{~m}$ & $<36 \mathrm{~d}$ & $\begin{array}{l}\text { 52d of Mutian X: } \\
7 \mathrm{~d} \text { at } 160 \mathrm{mg} / \mathrm{kg}, \\
\text { reduced to } \\
120 \mathrm{mg} / \mathrm{kg} \text { then } \\
80 \mathrm{mg} / \mathrm{kg} ; \\
\text { followed by } 1 \mathrm{x} \\
10^{5} \text { units of } \\
\text { rFelFN- } \omega \text { per os } \\
\text { q24h. Also } \\
\text { cobalamin } \\
\text { (Cobalaplex); } \\
\text { amoxycillin and } \\
\text { clavulanic acid } \\
\text { (Synulox, Zoetis } \\
\text { UK Ltd), } \\
\text { mirtazapine } \\
\text { (Summit } \\
\text { Veterinary } \\
\text { Pharmaceuticals, } \\
\text { UK); cobalamin } \\
\text { (Cobalaplex); } \\
\text { SAMe } \\
\text { (Denamarin). }\end{array}$ & $\begin{array}{l}\text { No: meloxicam } \\
\text { instead }\end{array}$ \\
\hline 18 & Wish & $\begin{array}{l}\text { Non- } \\
\text { effusive }\end{array}$ & $\begin{array}{l}\text { IHC of MLN } \\
\text { biopsy pos RT- } \\
\text { PCR (negative } \\
\text { for mutations) }\end{array}$ & $>5 \mathrm{~m}$ & $\begin{array}{l}<13 d \text { but } \\
\text { no initial } \\
\text { test so no } \\
\text { proof it } \\
\text { was ever } \\
\text { raised }\end{array}$ & $\begin{array}{l}29 \mathrm{~d} \text { of Mutian X: } \\
7 \mathrm{~d} \text { at } 160 \mathrm{mg} / \mathrm{kg} \\
\text { and } 22 \mathrm{~d} \text { at } \\
80 \mathrm{mg} / \mathrm{kg} ; \\
\text { followed by } 1 \mathrm{x} \\
10^{5} \text { units of } \\
\text { rFelFN- } \omega \text { per os } \\
\text { q24h. Cobalamin } \\
\text { (Cobalaplex); } \\
\text { Denamarin. }\end{array}$ & For 7d only \\
\hline 19 & Mike & $\begin{array}{l}\text { Non- } \\
\text { effusive } \\
\text { (colonic) }\end{array}$ & Biopsy & $>2.5 y$ & $\begin{array}{l}\text { unknown: } \\
\text { AGP } \\
\text { tested } \\
\text { only once } \\
\text { after 1y } \\
\text { of } \\
\text { treatment }\end{array}$ & $\begin{array}{l}1 \times 10^{5} \text { units } \\
\text { rFelFN- } \omega \text { per os } \\
\text { q24h for } 5 \mathrm{~m} \text {, } \\
\text { meloxicam and } \\
\text { Pl, followed by } \\
12 w \text { of GS- } \\
441524 \text { (DC } \\
\text { Chemicals, USA), } \\
\text { followed by } 1 \mathrm{x} \\
10^{5} \text { units of } \\
\text { rFelFN- } \omega \text { per os } \\
\text { q24 h, and PI. }\end{array}$ & No \\
\hline 20. & Chynah & $\begin{array}{l}\text { Effusive } \\
\text { (pleural } \\
\text { effusion) }\end{array}$ & $\mathrm{RT}^{\mathrm{PCR}} \mathrm{C}_{T} 33$ & $>7 \mathrm{~m}$ & $\begin{array}{l}\text { remained } \\
\text { raised > } \\
3 \mathrm{~m}\end{array}$ & $\begin{array}{l}\text { rFelFN- } \omega 1 \mathrm{MU} / \mathrm{kg} \\
\text { s/c q48h, } \\
\text { reducing to twice } \\
\text { weekly for } 5 \mathrm{~m} \text {. } \\
\text { Repeated } \\
\text { drainage of } \\
\text { effusion. }\end{array}$ & $\begin{array}{l}2.5 \mathrm{mg} / \text { cat q } 24 \mathrm{~h} \\
\text { sliding doses }\end{array}$ \\
\hline
\end{tabular}




\begin{tabular}{|c|c|c|c|c|c|c|c|}
\hline & Cat & $\begin{array}{l}\text { FIP } \\
\text { presentation }\end{array}$ & $\begin{array}{l}\text { How FIP } \\
\text { diagnosed }\end{array}$ & $\begin{array}{l}\text { Survival } \\
\text { in years } \\
\text { or months }\end{array}$ & $\begin{array}{l}\text { Time to } \\
\text { normal } \\
\text { AGP }\end{array}$ & Treatments & Prednisolone \\
\hline 21 & Tabitha & $\begin{array}{l}\text { Non- } \\
\text { effusive }\end{array}$ & FIP profile & $>7 m$ & $<29 \mathrm{~d}$ & $\begin{array}{l}58 \mathrm{~d} \text { of Mutian } \mathrm{X} \\
\text { starting at } \\
160 \mathrm{mg} / \mathrm{kg} \text { for } 8 \\
\text { days, then } \\
120 \mathrm{mg} / \mathrm{kg} \text { for } \\
20 \mathrm{~d} \text { then } \\
80 \mathrm{mg} / \mathrm{kg} \text {; } \\
\text { followed by } 1 \mathrm{x} \\
10^{5} \text { units of } \\
\text { rFelFN- } \omega \text { per os } \\
\text { q24h. Pro-kolin } \\
\text { enterogenic } \\
\text { probiotics. }\end{array}$ & No: meloxicam \\
\hline 22 & Harry & $\begin{array}{l}\text { Small } \\
\text { amount of } \\
\text { ascites and } \\
\text { enlarged } \\
\text { MLN }\end{array}$ & $\begin{array}{l}\text { FCoV RT-PCR } \\
\text { pos MLN. } \\
\text { Biopsy } \\
\text { inconclusive. }\end{array}$ & $>6 m$ & $<30 d$ & $\begin{array}{l}50 \mathrm{~d} \text { of Mutian } \mathrm{X} \text { : } \\
43 \mathrm{~d} \text { at } 80 \mathrm{mg} / \mathrm{kg} \\
\text { then } 7 \mathrm{~d} \text { at } \\
160 \mathrm{mg} / \mathrm{kg} ; \\
\text { followed by } 1 \mathrm{x} \\
10^{5} \text { units of } \\
\text { rFelFN- } \omega \text { per os } \\
\text { q24h. Cobalamin, } \\
\text { SAMe. }\end{array}$ & $\begin{array}{l}5.0 \mathrm{mg} \text { / cat q12h } \\
\text { for } 5 \mathrm{~d}\end{array}$ \\
\hline 23 & $\begin{array}{l}\text { Mr } \\
\text { Twinkles }\end{array}$ & $\begin{array}{l}\text { Effusive } \\
\text { (ascites) }\end{array}$ & FIP profile & $>8 m$ & $<117 d$ & $\begin{array}{l}7 \mathrm{mg} / \mathrm{kg} \text { Spark } \\
\text { Jones et al, } 202144 \\
\text { injection s/c for } \\
10 \mathrm{~d} \text {, followed by } \\
4 \text { Spark } 5 \mathrm{mg} \\
\text { tablets (i.e. } \\
7 \mathrm{mg} / \mathrm{kg} \text { ) per os } \\
\text { q24h for } 84 \mathrm{~d} . \\
\text { Cobalamin } \\
\text { (Cobalaplex). } \\
\text { Denamarin. }\end{array}$ & No \\
\hline 24 & Tyra & $\begin{array}{l}\text { Non- } \\
\text { effusive } \\
\text { (uveitis) }\end{array}$ & FIP profile & $>10 m$ & $<68 d$ & $\begin{array}{l}\text { Pine and Lucky } \\
\text { Jones et al, } 202144 \\
\text { injections (12- } \\
14 \mathrm{mg} / \mathrm{kg}) \text { : Pine } \\
\text { for } 4 \mathrm{~d}, \text { Lucky } \\
\text { injections for } 68 \mathrm{~d} \text {. } \\
\text { Then } 9 \text { Lucky red } \\
\text { pills per os q24h } \\
\text { for } 13 \mathrm{~d} \text {. }\end{array}$ & No \\
\hline 25 & Munchie & $\begin{array}{l}\text { Effusive } \\
\text { (ascites) }\end{array}$ & FIP profile & $>1.5 y$ & $<108 d$ & $\begin{array}{l}\text { Spark } 8 \mathrm{mg} / \mathrm{kg} \text { per } \\
\text { os q24h for } 84 \mathrm{~d} \text {. }\end{array}$ & No \\
\hline 26 & Edward & $\begin{array}{l}\text { Effusive } \\
\text { (pleural } \\
\text { effusion) }\end{array}$ & $\begin{array}{l}\text { FIP profile } \\
\text { incuding } \\
\text { cytology }\end{array}$ & $\begin{array}{l}10 \mathrm{~m} \\
(+\mathrm{RTA})\end{array}$ & $<7 \mathrm{~m}$ & $\begin{array}{l}\text { rFelFN- } \omega 1 \mathrm{MU} / \mathrm{kg} \\
\text { s/c q48h, } \\
\text { meloxicam per os } \\
\text { q24h. }\end{array}$ & No \\
\hline \multicolumn{8}{|c|}{$>$ indicates that survival was over this period of time + indicates death } \\
\hline \multicolumn{8}{|c|}{ AGP - alpha-1 acid glycoprotein } \\
\hline \multicolumn{8}{|c|}{$\mathrm{C}_{T-\text { Cycle threshold }}$} \\
\hline
\end{tabular}




\begin{tabular}{|c|c|c|c|c|c|c|}
\hline Cat & $\begin{array}{l}\text { FIP } \\
\text { presentation }\end{array}$ & $\begin{array}{l}\text { How FIP } \\
\text { diagnosed }\end{array}$ & $\begin{array}{l}\text { Survival } \\
\text { in years } \\
\text { or months }\end{array}$ & $\begin{array}{l}\text { Time to } \\
\text { normal } \\
\text { AGP }\end{array}$ & Treatments & Prednisolone \\
\hline \multicolumn{7}{|c|}{ FNA - fine needle aspirate } \\
\hline \multicolumn{7}{|c|}{ GC376 - a 3c-like protease inhibitor ${ }^{18 .(P e d e r s e n ~ e t ~ a l, ~ 2018) ~}$} \\
\hline \multicolumn{7}{|c|}{ GS-441524 - an injectable nucleoside analogue ${ }^{19 .(P e d e r s e n ~ e t ~ a l, ~ 2019) ~}$} \\
\hline \multicolumn{7}{|l|}{$\mathrm{h}$ - hours } \\
\hline \multicolumn{7}{|c|}{$\mathrm{m}$ - months } \\
\hline \multicolumn{7}{|l|}{ w - weeks } \\
\hline \multicolumn{7}{|l|}{$y$ - years } \\
\hline \multicolumn{7}{|c|}{$\mathrm{IHC}$ - immunohistochemistry } \\
\hline \multicolumn{7}{|c|}{ MLN - mesenteric lymph node } \\
\hline \multicolumn{7}{|c|}{ MU - million units } \\
\hline \multicolumn{7}{|c|}{$\begin{array}{l}\text { Mutian - Mutian Xraphconn (Nantong Mutian Biotechnology Co. Ltd. China), an adenosine nucleoside analogue }{ }^{33} \\
\text { (Addie et al, 2020) }\end{array}$} \\
\hline \multicolumn{7}{|c|}{$\mathrm{PI}$ - polyprenyl immunostimulant (PI, Vetlmmune, Sass \& Sass, USA) } \\
\hline \multicolumn{7}{|c|}{ Pos - positive } \\
\hline \multicolumn{7}{|c|}{ rFelFN- $\omega$ - recombinant feline interferon omega (Virbac, France) } \\
\hline \multicolumn{7}{|c|}{ RTA - road traffic accident } \\
\hline \multicolumn{7}{|c|}{ RT-PCR - reverse transcriptase polymerase chain reaction } \\
\hline \multicolumn{7}{|c|}{ SAMe - S-adenosyl-L-methionine (Denamarin, Nutramax Laboratories, Inc, USA) } \\
\hline s/c-subcl & usly & & & & & \\
\hline
\end{tabular}


Table 2

How FIP was diagnosed, survival time and treatment details of 16 cats who went into remission or died

\begin{tabular}{|c|c|c|c|c|c|c|}
\hline Ref & Cat & $\begin{array}{l}\text { FIP } \\
\text { presentation }\end{array}$ & $\begin{array}{l}\text { How FIP } \\
\text { diagnosed }\end{array}$ & $\begin{array}{l}\text { Survival } \\
\text { in } \\
\text { months }\end{array}$ & Treatments & Prednisolone \\
\hline 1 & Yrael & Effusive & $\begin{array}{l}\mathrm{C}_{T} 32 \mathrm{RT}- \\
\text { qPCR on } \\
\text { effusion }\end{array}$ & $1.5+$ & $\begin{array}{l}\text { Coconut oil and draining the } \\
\text { effusion. }\end{array}$ & Unknown \\
\hline 2 & $\begin{array}{l}\text { Charlie } \\
\text { Chaplin }\end{array}$ & $\begin{array}{l}\text { Non- } \\
\text { effusive }\end{array}$ & $\begin{array}{l}\mathrm{C}_{T} 25 \mathrm{MLN} \\
\text { FNA then } \\
\text { histopath }\end{array}$ & $1.5+$ & $\begin{array}{l}1 \times 10^{5} \mathrm{rFelFN}-\omega \text { per os } \mathrm{q} 24 \mathrm{~h} \text {. } \\
\text { Clindamycin for toxoplasmosis co- } \\
\text { infection. }\end{array}$ & ] \\
\hline 3 & Smokey & $\begin{array}{l}\text { Non- } \\
\text { effusive }\end{array}$ & $\begin{array}{l}\mathrm{C}_{T} 28 \mathrm{MLN} \\
\text { FNA RT- } \\
\text { qPCR, then } \\
\text { histopath }\end{array}$ & $1.8+$ & Adipose stem cell therapy. & Unknown \\
\hline 4 & Rowley & $\begin{array}{l}\text { Effusive } \\
\text { (ascites) } \\
\text { then non- } \\
\text { effusive } \\
\text { (uveitis, } \\
\text { finally } \\
\text { severe } \\
\text { anaemia) }\end{array}$ & $\begin{array}{l}\mathrm{C}_{T} 28.4 \text { on } \\
\text { effusion } \\
\text { then } \\
\text { histopath }\end{array}$ & $2.25+$ & $\begin{array}{l}1 \mathrm{MU} / \mathrm{kg} \text { rFelFN- } \omega \text { s/c q } 48 \mathrm{~h} \text { resulted } \\
\text { in resolution of his ascites then } 1 \mathrm{x} \\
10^{5} \text { units per os q } 24 \mathrm{~h} \text {. Mirtazapine } 3 \\
\text { times. Anti-TNF-alpha monoclonal } \\
\text { antibody infliximab, (Remicade } \AA \text {, } \\
\mathrm{MSD} \text {, UK) } 4 \mathrm{mg} / \mathrm{kg} \text { in a } 0.9 \% \text { saline } \\
\text { infusion over a } 4 \mathrm{~h} \text { period. Type B } \\
\text { blood transfusion. }\end{array}$ & $\begin{array}{l}5 \mathrm{mg} q 12 \mathrm{~h}, \\
\text { dose not } \\
\text { reduced }\end{array}$ \\
\hline 5 & Claude & $\begin{array}{l}\text { Effusive } \\
\text { (pleural } \\
\text { effusion } \\
\text { initially then } \\
\text { also } \\
\text { ascites) }\end{array}$ & $\begin{array}{l}\text { Partial FIP } \\
\text { profile }\end{array}$ & $3.0+$ & $\begin{array}{l}1 \mathrm{MU} / \mathrm{kg} \text { rFelFN-w s/c q48h. } \\
\text { Thoracentesis. }\end{array}$ & $\begin{array}{l}\bigotimes 1 \mathrm{mg} / \mathrm{kg} \\
\text { sliding } \\
\text { doses }\end{array}$ \\
\hline 6 & Alfie & $\begin{array}{l}\text { Non- } \\
\text { effusive } \\
\text { (chronic } \\
\text { diarrhoea, } \\
\text { enlarged } \\
\text { MLN) }\end{array}$ & $\begin{array}{l}\text { Histopath. } \\
\mathrm{C}_{T} 31 \mathrm{MLN} \\
\text { FNA RT- } \\
\text { qPCR } \\
\mathrm{C}_{T} 29 \\
\text { faeces }\end{array}$ & $3.5+$ & $\begin{array}{l}\text { rFelFN- } \omega \text { given per os, but dose not } \\
\text { recorded. Fortiflora probiotics } \\
\text { (Purina). }\end{array}$ & $\begin{array}{l}\otimes \text { dose not } \\
\text { recorded }\end{array}$ \\
\hline 7 & Holly & $\begin{array}{l}\text { Non- } \\
\text { effusive to } \\
\text { effusive } \\
\text { ascites }\end{array}$ & $\begin{array}{l}\text { mRNA and } \\
\text { 3'URT RT- } \\
\text { PCR pos }\end{array}$ & $4 \dagger$ & $\begin{array}{l}5 \times 10^{4} \text { rFelFN-w per os. Colloidal } \\
\text { silver (dose unknown). }\end{array}$ & Unknown \\
\hline 8 & Bugsy & $\begin{array}{l}\text { Non- } \\
\text { effusive }\end{array}$ & FIP Profile & $6+$ & $\begin{array}{l}1 \mathrm{MU} / \mathrm{kg} \text { rFelFN- } \omega \mathrm{s} / \mathrm{c} \mathrm{q} 48 \mathrm{~h} \text { then } 1 \mathrm{x} \\
10^{5} \text { units per os } \mathrm{q} 24 \mathrm{~h} .\end{array}$ & $\nabla 5 \mathrm{mg}$ eod \\
\hline 9 & Daisy & $\begin{array}{l}\text { Non- } \\
\text { effusive }\end{array}$ & $\begin{array}{l}\mathrm{C}_{T} 27 \mathrm{RT}- \\
\text { qPCR MLN } \\
\text { FNA }\end{array}$ & $5+$ & $\begin{array}{l}1 \mathrm{MU} / \mathrm{kg} \text { rFelFN- } \omega \mathrm{s} / \mathrm{c} \mathrm{q} 48 \mathrm{~h} \text { then } \\
\text { once a week, then } 5 \times 10^{4} \text { units } \\
\text { rFelFN- } \omega \text { per os } \mathrm{q} 24 \mathrm{~h} \text {. }\end{array}$ & $\begin{array}{l}\otimes \text { higher } \\
\text { dose } \\
\text { reduced to } \\
0.5 \mathrm{mg} / \mathrm{kg} \\
\text { q24h after } \\
14 \mathrm{~d}\end{array}$ \\
\hline 10 & Levi & $\begin{array}{l}\text { Effusive } \\
\text { (ascites) }\end{array}$ & $\begin{array}{l}\mathrm{C}_{T} 33 \mathrm{RT}- \\
\text { qPCR on } \\
\text { ascites }\end{array}$ & $5.5 \dagger$ & $\begin{array}{l}1 \mathrm{MU} / \mathrm{kg} \text { rFelFN- } \omega \mathrm{s} / \mathrm{c} \text { twice a week. } \\
\text { Ascites drained. }\end{array}$ & $\begin{array}{l}\text { \dose not } \\
\text { recorded }\end{array}$ \\
\hline
\end{tabular}




\begin{tabular}{|c|c|c|c|c|c|c|}
\hline Ref & Cat & $\begin{array}{l}\text { FIP } \\
\text { presentation }\end{array}$ & $\begin{array}{l}\text { How FIP } \\
\text { diagnosed }\end{array}$ & $\begin{array}{l}\text { Survival } \\
\text { in } \\
\text { months }\end{array}$ & Treatments & Prednisolone \\
\hline 11 & Roxanne & $\begin{array}{l}\text { Non- } \\
\text { effusive and } \\
\text { FGS }\end{array}$ & $\begin{array}{l}\mathrm{C}_{T} 42 \\
\text { pbmcs } \\
\mathrm{C}_{T} 23 \\
\text { faeces }\end{array}$ & $11+$ & $\begin{array}{l}1 \mathrm{MU} / \mathrm{kg} \text { rFelFN- } \omega \mathrm{s} / \mathrm{c} \text { twice weekly. } \\
\text { Clindamycin (Antirobe, Zoetis, UK), } \\
\text { meloxicam. }\end{array}$ & $\begin{array}{l}\text { No: } \\
\text { meloxicam }\end{array}$ \\
\hline 12 & Pip & $\begin{array}{l}\text { Non- } \\
\text { effusive }\end{array}$ & $\begin{array}{l}\text { Very low } \\
\text { positive }\left(C_{T}\right. \\
40) \text { RT-qPCR } \\
\text { pbmcs } \\
\text { twice Feb } \\
2005\end{array}$ & $>8$ & $5 \times 10^{4}$ units rFelFN- $\omega$ per os $\mathrm{q} 24 \mathrm{~h}$ & $\begin{array}{l}\text { \dose not } \\
\text { recorded }\end{array}$ \\
\hline 13 & Pharaoh & $\begin{array}{l}\text { Non- } \\
\text { effusive }\end{array}$ & $\begin{array}{l}\mathrm{C}_{T} 27 \mathrm{MLN} \\
\text { biopsy } \\
\text { inconclusive }\end{array}$ & $8+$ & Adipose stem cell therapy. & Unknown \\
\hline 14 & Maximus & $\begin{array}{l}\text { Non- } \\
\text { effusive }\end{array}$ & $\begin{array}{l}\text { Positive RT- } \\
\text { PCR MLN } \\
\text { FNA }\end{array}$ & $14+$ & $1 \mathrm{MU} / \mathrm{kg}$ rFelFN-w s/c q48h & $\begin{array}{l}5 \mathrm{mg} / \mathrm{cat} \\
\mathrm{q} 24 \mathrm{~h}\end{array}$ \\
\hline 15 & Ragamuffin & $\begin{array}{l}\text { Non- } \\
\text { effusive }\end{array}$ & $\begin{array}{l}\text { Biopsy MLN } \\
\text { \& intestine } \\
\text { non-spec }\end{array}$ & $>60$ & $\begin{array}{l}1 \mathrm{MU} / \mathrm{kg} \text { rFelFN- } \omega \mathrm{s} / \mathrm{c} \text { q } 48 \mathrm{~h} \text { then } 1 \mathrm{x} \\
10^{5} \text { units per os q } 24 \mathrm{~h} \text { then } 1 \mathrm{MU} / \mathrm{kg} \\
\text { rFelFN- } \omega \text { twice a week }\end{array}$ & $\begin{array}{l}\text { QSliding } \\
\text { doses }\end{array}$ \\
\hline 16 & Tinkerbell & $\begin{array}{l}\text { Non- } \\
\text { effusive }\end{array}$ & $\begin{array}{l}\text { FIP Profile } \\
\mathrm{C}_{T} 31-34 \\
\text { faeces }\end{array}$ & $>36$ & $\begin{array}{l}1 \mathrm{MU} / \mathrm{kg} \text { rFelFN- } \omega \mathrm{s} / \mathrm{c} \mathrm{q} 48 \mathrm{~h} \text { then } 1 \mathrm{x} \\
10^{5} \text { units per os } \mathrm{q} 24 \mathrm{~h} . \mathrm{PI} \text {. } \\
\text { Mirtazapine } 2 \mathrm{mg} / \text { cat q48h. }\end{array}$ & $\begin{array}{l}\otimes \text { For over 1y } \\
\text { at } 2 \mathrm{mg} / \mathrm{kg}\end{array}$ \\
\hline \multicolumn{7}{|c|}{$>$ indicates that survival was over this period of time + indicates death } \\
\hline \multicolumn{7}{|c|}{ AGP - alpha-1 acid glycoprotein } \\
\hline \multicolumn{7}{|c|}{$\mathrm{C}_{T-}$ Cycle threshold } \\
\hline \multicolumn{7}{|c|}{ FCoV - feline coronavirus } \\
\hline \multicolumn{7}{|c|}{ FGS - feline chronic gingivostomatitis } \\
\hline \multicolumn{7}{|c|}{ FNA - fine needle aspirate } \\
\hline \multicolumn{7}{|c|}{ GC376 - a 3c-like protease inhibitor 18.( (Pedersen et al, 2018) } \\
\hline \multicolumn{7}{|c|}{ GS-441524 - an injectable nucleoside analogue ${ }^{19 .}$ Pedersen et al, 2019) } \\
\hline \multicolumn{7}{|c|}{$\mathrm{h}-$ hours } \\
\hline \multicolumn{7}{|c|}{$\mathrm{m}$ - months } \\
\hline \multicolumn{7}{|c|}{ w - weeks } \\
\hline \multicolumn{7}{|c|}{$y$ - years } \\
\hline \multicolumn{7}{|c|}{ IHC - immunohistochemistry } \\
\hline \multicolumn{7}{|c|}{ MLN - mesenteric lymph node } \\
\hline MU & million units & & & & & \\
\hline
\end{tabular}




\begin{tabular}{llllll} 
Ref Cat & $\begin{array}{l}\text { FIP } \\
\text { presentation }\end{array}$ & $\begin{array}{l}\text { How FIP } \\
\text { diagnosed }\end{array}$ & $\begin{array}{l}\text { Survival } \\
\text { in } \\
\text { months }\end{array}$ & Treatments & Prednisolone \\
\hline
\end{tabular}

Mutian - Mutian Xraphconn (Nantong Mutian Biotechnology Co. Ltd. China), an adenosine nucleoside analogue 32 (Addie et al, 2020)

$\mathrm{PI}$ - polyprenyl immunostimulant (PI, VetImmune, Sass \& Sass, USA)

Pos - positive

rFelFN- $\omega$ - recombinant feline interferon omega (Virbac, France)

RT-PCR - reverse transcriptase polymerase chain reaction

SAMe - S-adenosyl-L-methionine (Denamarin, Nutramax Laboratories, Inc, USA)

s/c - subcutaneously

Veterinary surgeons seek a criterion by which to be assured that the cat has recovered in order to know when to cease treatment. Markers for poor prognosis in FIP have been well defined ${ }^{10}$, yet no guidelines have been established to define a recovery from FIP, probably because, until recently, it was widely assumed that recovery from FIP could not occur.

No survival time point after which a cat can be said to have recovered from FIP has been established. In their report of their seminal study of successful treatment of FIP with an antiviral drug, Pedersen et al wrote: "It raises the question of how long remission must be sustained to declare the disease cured, rather than in a sustained remission." 18 The purpose of this paper was to attempt to answer that question; but rather than duration of survival, we found that a return of elevated AGP to normal levels is a rapid and definitive marker for recovery from FIP.

\section{Materials And Methods}

Selection of cases. This study retrospectively evaluated the medical records of 42 cats diagnosed with FIP between January 2004 and March 2021. Cats were selected for this study if sequential laboratory test results were available, including AGP measurements, a reasonable certainty that the FIP diagnosis was correct, and whose survival outcomes were known.

FIP diagnosis. The data presented here were collected over almost two decades. As new tests became available they were applied to the cases; consequently, details of FIP diagnosis of individual cats were not uniform. While demonstration of FCoV in biopsy or post mortem material by immunohistochemistry is deemed the gold standard for FIP diagnosis, for practical purposes it is generally accepted that a positive FCoV RNA ${ }^{20,21}$, test by RT-PCR on effusion ${ }^{18,19,20,22,23,24,25}$, peripheral blood mononuclear cells (pbmcs) ${ }^{26,27}$, or lymph node fine needle aspirate (FNA) ${ }^{28}$ is sufficiently indicative of FIP to commence treatment ${ }^{18}$. Where mutations M1058L or S1060A were also positive, this was considered additional evidence for FIP diagnosis ${ }^{29,30}$, although it has been argued that these mutations are only indicative of systemic spread of the virus ${ }^{31}$.

As shown in Tables 1 and 2, diagnosis of FIP was by histopathology $(n=13)$, of which two cases had positive immunohistochemistry (IHC). However, histopathology of biopsies was inconclusive in five cases and was reported nonspecifically as "pyogranulomatous inflammation."

FIP diagnosis was by detection of FCoV RNA as demonstrated by RT-PCR in effusions ( $\mathrm{n}=10)$, or mesenteric lymph node $(M L N) F^{28}(n=10)$; messenger RNA (mRNA) ${ }^{26,27}$ or $3^{\prime} U T R R^{20,21}$ in pbmc $(n=4)$. One of two cats in which the mutation tests 29,30 were performed was positive for the M1058L mutation, and neither cat was positive for the S1060A mutation. 
Some cats fulfilled multiple diagnostic criteria for FIP: seven cats had both positive FCoV RT-PCR tests and histopathology. All 42 cats were positive for at least some components of an FIP profile consisting of typical clinical signs, FCoV antibody titre, raised AGP, hyperglobulinaemia, anaemia, lymphopenia (Tables 3 and 4). In 11 cats the FIP profile was the sole evidence for its diagnosis, although a positive response to an anti-coronavirus drug was thought to corroborate the FIP diagnosis. AGP was raised in all cats except two where a pre-treatment test was not done. It has been documented that raised AGP was more sensitive than histopathology for diagnosing FIP in challenging cases $^{13}$. 
Table 3

FIP score before and after treatment in the recovered group

\begin{tabular}{|c|c|c|c|c|c|c|c|c|}
\hline & \multirow[t]{2}{*}{ Cat } & \multirow[t]{2}{*}{ Clinical signs } & \multirow{2}{*}{$\begin{array}{l}\text { FCoV } \\
\text { antibody } \\
\text { titre (interval } \\
\text { first to last) }\end{array}$} & \multirow{2}{*}{$\begin{array}{l}\text { Anaemia } \\
\text { (Hct \%) }\end{array}$} & \multirow{2}{*}{$\begin{array}{l}\text { Lympho- } \\
\text { penia }\end{array}$} & \multirow{2}{*}{$\begin{array}{l}\text { Hypergamma- } \\
\text { globulinaemia }\end{array}$} & \multicolumn{2}{|l|}{ Score } \\
\hline & & & & & & & Before & After \\
\hline \multirow[t]{3}{*}{1} & \multirow[t]{3}{*}{ Basil 1} & \multirow[t]{3}{*}{ Icterus } & \multirow{3}{*}{$\begin{array}{l}1280 / 10 \\
(13 m)\end{array}$} & $\nabla / \otimes$ & \multirow[t]{3}{*}{$\nabla / \otimes$} & \multirow[t]{3}{*}{ 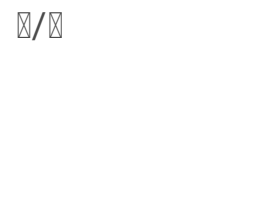 } & \multirow[t]{3}{*}{5} & \multirow[t]{3}{*}{0} \\
\hline & & & & $28.2 / 40.9$ & & & & \\
\hline & & & & $(22 d)$ & & & & \\
\hline 2 & Boris & $\begin{array}{l}\text { Poor appetite, } \\
\text { diarrhoea }\end{array}$ & $\begin{array}{l}>1280 / 160 \\
(4 \mathrm{~m})\end{array}$ & ? & प & प & 2 & 1 \\
\hline 3 & Mars & Anorexia & $\begin{array}{l}>1280 / 320 \\
(18 m)\end{array}$ & ૫ & $\nabla / \nabla$ & $\nabla / \nabla$ & 4 & 1 \\
\hline 4 & Chester & $\begin{array}{l}\text { Pleural effusion, } \\
\text { pyrexia }\end{array}$ & $\begin{array}{l}>1280 / \\
1280(28 \mathrm{~m})\end{array}$ & $\nabla^{*}$ & प & $N A / \mathbb{B}$ & 2 & 1 \\
\hline 5 & Amy & $\begin{array}{l}\text { Weight loss, } \\
\text { pyrexia, tender } \\
\text { abdomen. Icteric } \\
\text { plasma. }\end{array}$ & $\begin{array}{l}1280 / 640 \\
(16 \mathrm{~m})\end{array}$ & प & $\nabla / \nabla$ & $\nabla / \bigotimes$ & 4 & 1 \\
\hline \multirow[t]{2}{*}{6} & \multirow[t]{2}{*}{ Brook } & \multirow[t]{2}{*}{ Recurring pyrexia } & $1280 / 0$ & $\nabla / \nabla$ & \multirow[t]{2}{*}{$\nabla / \nabla$} & $\nabla / \otimes$ & \multirow[t]{2}{*}{5} & \multirow[t]{2}{*}{1} \\
\hline & & & $(13 m)$ & $(33 d)$ & & $(57-51)$ & & \\
\hline 7 & Basil 2 & Ascites & $\begin{array}{l}>1280 / \\
>1280 \\
(27 m)\end{array}$ & $\nabla / \nabla$ & $\nabla / \nabla$ & $\nabla / \otimes$ & 5 & 1 \\
\hline 8 & Kitten 2 & $\begin{array}{l}\text { Ascites. Relapse: } \\
\text { painful tail, } \\
\text { ataxia, seizures. } \\
\text { Full recovery. }\end{array}$ & $\begin{array}{l}>1280 / 640 \\
(8 \mathrm{~m})\end{array}$ & ? & प & $\nabla / \nabla$ & 5 & 1 \\
\hline 9 & Skywise & $\begin{array}{l}\text { Weight loss, } \\
\text { uveitis }\end{array}$ & $\begin{array}{l}>10,240 \\
/ 640(9 \mathrm{~m})\end{array}$ & $\nabla / \otimes(12 d)$ & $\nabla / \nabla$ & $\nabla / \bigotimes$ & 5 & 0 \\
\hline \multirow[t]{2}{*}{10} & \multirow[t]{2}{*}{ Betsy } & \multirow{2}{*}{$\begin{array}{l}\text { Ascites, profound } \\
\text { anaemia, } \\
\text { underweight: BCS } \\
2 / 9\end{array}$} & \multirow{2}{*}{$\begin{array}{l}>1280 / \\
>1280(2 \mathrm{~m})\end{array}$} & 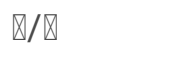 & \multirow[t]{2}{*}{$\nabla / \nabla$} & \multirow[t]{2}{*}{ Q } & \multirow[t]{2}{*}{4} & \multirow[t]{2}{*}{1} \\
\hline & & & & $\begin{array}{l}14.0 / 39.4 \\
(20 d)\end{array}$ & & & & \\
\hline \multirow[t]{2}{*}{11} & \multirow[t]{2}{*}{ Dante } & \multirow{2}{*}{$\begin{array}{l}\text { Vomiting and } \\
\text { diarrhoea }\end{array}$} & \multirow{2}{*}{$\begin{array}{l}1280 / \\
>1280(2 \mathrm{~m})\end{array}$} & \multirow[t]{2}{*}{$\nabla / \nabla$} & \multirow[t]{2}{*}{ प } & 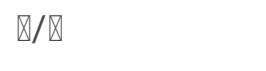 & 3 & 1 \\
\hline & & & & & & $(68-53)$ & & \\
\hline
\end{tabular}

NA - not available WNL - within normal limits

Hct - haematocrit

\present $\nabla$ - absent

* Only one result just under the Hct cut-off of $30 \%$ (i.e. $29 \%$ on one occasion)

This table shows the clinical signs before treatment; FCoV antibody titres first and most recent, with the interval in parenthesis; Hct before and after with the time taken for anaemia to resolve; lymphopenia; hyperglobulinaemia, showing reduction in parenthesis if globulins had not returned to normal $(<45 \mathrm{~g} / \mathrm{l})$. 


\begin{tabular}{|c|c|c|c|c|c|c|c|c|}
\hline & \multirow[t]{2}{*}{ Cat } & \multirow[t]{2}{*}{ Clinical signs } & \multirow{2}{*}{$\begin{array}{l}\text { FCoV } \\
\text { antibody } \\
\text { titre (interval } \\
\text { first to last) }\end{array}$} & \multirow{2}{*}{$\begin{array}{l}\text { Anaemia } \\
\text { (Hct \%) }\end{array}$} & \multirow{2}{*}{$\begin{array}{l}\text { Lympho- } \\
\text { penia }\end{array}$} & \multirow{2}{*}{$\begin{array}{l}\text { Hypergamma- } \\
\text { globulinaemia }\end{array}$} & \multicolumn{2}{|l|}{ Score } \\
\hline & & & & & & & Before & After \\
\hline 12 & Elmo & $\begin{array}{l}\text { Quiet purr, poor } \\
\text { appetite, listless. } \\
\text { Pyrexia, } \\
\text { mesenteric lymph } \\
\text { node } \\
\text { enlargement, } \\
\text { anaemia }\end{array}$ & $\begin{array}{l}>10.240 / 0 \\
(8 \mathrm{~m})\end{array}$ & $\nabla / \square(31 d)$ & प & 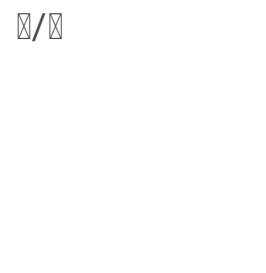 & 4 & 0 \\
\hline 13 & Lyra & Ascites & $\begin{array}{l}>10240 / \\
2560(8 \mathrm{~m})\end{array}$ & $\nabla / \otimes(47 d)$ & 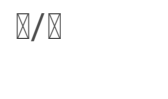 & $\nabla / \mathbb{\Delta}$ & 5 & 0 \\
\hline 14 & Molly & $\begin{array}{l}\text { Uveitis, chronic } \\
\text { diarrhoea: carrier } \\
\text { cat shed virus in } \\
\text { faeces }>23 \mathrm{~m}\end{array}$ & $\begin{array}{l}>1280 / \\
1280(15 \mathrm{~m})\end{array}$ & प & \/ه (low) & $\nabla / \otimes$ & 3 & 2 \\
\hline 15 & Bea & $\begin{array}{l}\text { Ascites, sudden } \\
\text { weakness in } \\
\text { limbs. } \\
\text { Trichobezoar } \\
\text { caused vomiting. }\end{array}$ & $\begin{array}{l}>1280 / 640 \\
(11 \mathrm{~m})\end{array}$ & $\nabla / \nabla(26 d)$ & प & $\begin{array}{l}\otimes / \bigotimes \\
(78-53)\end{array}$ & 4 & 1 \\
\hline 16 & Buddie & Ascites & $\begin{array}{l}>1280 \\
/ 1280 \\
(7 \mathrm{~m})\end{array}$ & $\nabla / \nabla(26 d)$ & $\begin{array}{l}2 \text { of } 7 \\
\text { samples }\end{array}$ & $\begin{array}{l}\varangle / \otimes \\
(106-74)\end{array}$ & 4 & 1 \\
\hline 17 & Nelson & $\begin{array}{l}\text { Dull, reduced } \\
\text { appetite, enlarged } \\
\text { MLN, ascites after } \\
\text { biopsy }\end{array}$ & $\begin{array}{l}640 />1280 \\
(6 \mathrm{~m})\end{array}$ & ? & प & $\nabla / \otimes$ & 3 & 1 \\
\hline 18 & Wish & $\begin{array}{l}\text { Enlarged MLN, } \\
\text { chronic diarrhoea }\end{array}$ & $\begin{array}{l}>1280 / \text { not } \\
\text { re-tested yet }\end{array}$ & ( & 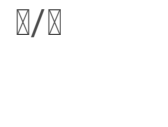 & $\begin{array}{l}\otimes / \square \\
(85-51)\end{array}$ & 4 & 2 \\
\hline 19 & Mike & $\begin{array}{l}\text { Chronic diarrhoea, } \\
\text { haematochezia, } \\
\text { continuous virus } \\
\text { shedding two } \\
\text { years later } \\
\text { although cat is } \\
\text { well }\end{array}$ & $\begin{array}{l}>1280 / \text { not } \\
\text { re-tested }\end{array}$ & 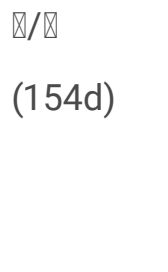 & $\nabla / \nabla$ & $\nabla / \otimes$ & 5 & $\begin{array}{l}1 \text { or } \\
2 ?\end{array}$ \\
\hline 20 & Chynah & $\begin{array}{l}\text { Cough, } \\
\text { pneumonia, nasal } \\
\text { discharge, } \\
\text { dyspnoea, pleural } \\
\text { effusion }\end{array}$ & $\begin{array}{l}1280 / \\
>1280(8 m)\end{array}$ & NA & NA & $\nabla / \otimes$ & 3 & 1 \\
\hline
\end{tabular}

NA - not available WNL - within normal limits

Hct - haematocrit

\present $\nabla$ - absent

* Only one result just under the Hct cut-off of $30 \%$ (i.e. $29 \%$ on one occasion)

This table shows the clinical signs before treatment; FCoV antibody titres first and most recent, with the interval in parenthesis; Hct before and after with the time taken for anaemia to resolve; lymphopenia; hyperglobulinaemia, showing reduction in parenthesis if globulins had not returned to normal $(<45 \mathrm{~g} / \mathrm{l})$. 


\begin{tabular}{|c|c|c|c|c|c|c|c|c|}
\hline & \multirow[t]{2}{*}{ Cat } & \multirow[t]{2}{*}{ Clinical signs } & \multirow{2}{*}{$\begin{array}{l}\text { FCoV } \\
\text { antibody } \\
\text { titre (interval } \\
\text { first to last) }\end{array}$} & \multirow{2}{*}{$\begin{array}{l}\text { Anaemia } \\
\text { (Hct \%) }\end{array}$} & \multirow{2}{*}{$\begin{array}{l}\text { Lympho- } \\
\text { penia }\end{array}$} & \multirow{2}{*}{$\begin{array}{l}\text { Hypergamma- } \\
\text { globulinaemia }\end{array}$} & \multicolumn{2}{|l|}{ Score } \\
\hline & & & & & & & Before & After \\
\hline \multirow[t]{2}{*}{21} & Tabitha & $\begin{array}{l}\text { Underweight, } \\
\text { pyrexic, watery } \\
\text { diarrhoea, ataxic } \\
\text { episode, possible } \\
\text { uveitis. }\end{array}$ & $\begin{array}{l}>1280 / \text { not } \\
\text { re-tested }\end{array}$ & $\nabla^{*}$ & $\begin{array}{l}\otimes \text { (but initial } \\
\text { count low at } \\
1.89)\end{array}$ & $\nabla / \nabla$ & 3 & 2 \\
\hline & & & & & & $(88-59)$ & & \\
\hline \multirow[t]{2}{*}{22} & \multirow[t]{2}{*}{ Harry } & \multirow{2}{*}{$\begin{array}{l}\text { Small amount of } \\
\text { ascites and } \\
\text { enlarged MLN }\end{array}$} & \multirow{2}{*}{$\begin{array}{l}>1280 \\
/>1280 \\
(2 m)\end{array}$} & \multirow[t]{2}{*}{$\nabla / \otimes(30 d)$} & \multirow[t]{2}{*}{$\nabla / \nabla$} & \multirow{2}{*}{$\begin{array}{l}\mathbb{Q / \otimes} \\
(99-54)\end{array}$} & \multirow[t]{2}{*}{5} & \multirow[t]{2}{*}{2} \\
\hline & & & & & & & & \\
\hline 23 & $\begin{array}{l}\text { Mr } \\
\text { Twinkles }\end{array}$ & Ascites & $\begin{array}{l}>1280 / \\
1280(6 \mathrm{~m})\end{array}$ & $\nabla / \otimes(57 d)$ & $\nabla / \nabla$ & $\nabla / \nabla$ & 5 & 1 \\
\hline \multirow[t]{2}{*}{24} & \multirow[t]{2}{*}{ Tyra } & \multirow[t]{2}{*}{ Uveitis } & \multirow{2}{*}{$\begin{array}{l}>1280 / 320 \\
(5 \mathrm{~m})\end{array}$} & \multirow[t]{2}{*}{ प } & \multirow[t]{2}{*}{ प } & $\nabla / \nabla$ & 3 & 1 \\
\hline & & & & & & $(58-54)$ & & \\
\hline \multirow[t]{2}{*}{25} & \multirow[t]{2}{*}{ Munchie } & \multirow{2}{*}{$\begin{array}{l}\text { Ascites, pyrexia, } \\
\text { lethargy }\end{array}$} & \multirow{2}{*}{$\begin{array}{l}>1280 / \\
>1280(4 \mathrm{~m})\end{array}$} & \multirow[t]{2}{*}{ ૫ } & \multirow[t]{2}{*}{$\square$} & $\nabla / \mathbb{Q}$ & 3 & 1 \\
\hline & & & & & & $(51-38)$ & & \\
\hline \multirow[t]{2}{*}{26} & \multirow[t]{2}{*}{ Edward } & \multirow[t]{2}{*}{ Pleural effusion } & \multirow{2}{*}{$\begin{array}{l}>1280 / \\
>1280(7 \mathrm{~m})\end{array}$} & \multirow{2}{*}{$\begin{array}{l}8 \text { but } 1 \mathrm{st} \\
\text { count } 2 \mathrm{~m} \\
\text { into } \\
\text { treatment }\end{array}$} & \multirow{2}{*}{$\begin{array}{l}\otimes \text { but } 1 \mathrm{st} \\
\text { count } 2 \mathrm{~m} \\
\text { into } \\
\text { treatment }\end{array}$} & $\nabla / \nabla$ & 3 & 1 \\
\hline & & & & & & $(59-44)$ & & \\
\hline
\end{tabular}

NA - not available WNL - within normal limits

Hct - haematocrit

\present $\nabla$ - absent

* Only one result just under the Hct cut-off of $30 \%$ (i.e. $29 \%$ on one occasion)

This table shows the clinical signs before treatment; FCoV antibody titres first and most recent, with the interval in parenthesis; Hct before and after with the time taken for anaemia to resolve; lymphopenia; hyperglobulinaemia, showing reduction in parenthesis if globulins had not returned to normal $(<45 \mathrm{~g} / \mathrm{l})$. 


\begin{tabular}{|c|c|c|c|c|c|c|c|}
\hline \multirow[t]{2}{*}{ Cat } & \multirow[t]{2}{*}{ Clinical signs } & \multirow{2}{*}{$\begin{array}{l}\text { FCoV } \\
\text { antibody } \\
\text { titre (interval } \\
\text { first to last) }\end{array}$} & \multirow{2}{*}{$\begin{array}{l}\text { Anaemia } \\
\text { (Hct \%) }\end{array}$} & \multirow{2}{*}{$\begin{array}{l}\text { Lympho- } \\
\text { penia }\end{array}$} & \multirow{2}{*}{$\begin{array}{l}\text { Hypergamma- } \\
\text { globulinaemia }\end{array}$} & \multicolumn{2}{|l|}{ Score } \\
\hline & & & & & & Before & After \\
\hline \multirow[t]{2}{*}{ Total } & & \multirow{2}{*}{$\begin{array}{l}7 \text { of } 26 \\
\text { FCoV } \\
\text { antibody } \\
\text { titres } \\
\text { reduced } \\
\text { significantly }\end{array}$} & \multirow{2}{*}{$\begin{array}{l}12 \text { of } 25 \\
\text { cats } \\
\text { anaemic: } \\
\text { anaemia } \\
\text { resolved } \\
\text { in all }\end{array}$} & \multirow[t]{2}{*}{$\begin{array}{l}9 \text { of } 24 \text { cats } \\
\text { lymphopenic } \\
\text { on at least } \\
\text { one test }\end{array}$} & $\begin{array}{l}22 / 25 \text { cats } \\
\text { had raised } \\
\text { globulins, one } \\
\text { NA. }\end{array}$ & & \\
\hline & & & & & $\begin{array}{l}\text { Reduced in all } \\
22 \text { and to } \\
\text { WNL in } 15 \\
\text { cats. }\end{array}$ & & \\
\hline \multicolumn{8}{|c|}{ NA - not available WNL - within normal limits } \\
\hline \multicolumn{8}{|c|}{ Hct - haematocrit } \\
\hline \multicolumn{8}{|c|}{ 『present $\nabla$ - absent } \\
\hline \multicolumn{8}{|c|}{ * Only one result just under the Hct cut-off of $30 \%$ (i.e. $29 \%$ on one occasion) } \\
\hline \multicolumn{8}{|c|}{$\begin{array}{l}\text { This table shows the clinical signs before treatment; FCoV antibody titres first and most recent, with the interval in } \\
\text { parenthesis; Hct before and after with the time taken for anaemia to resolve; lymphopenia; hyperglobulinaemia, } \\
\text { showing reduction in parenthesis if globulins had not returned to normal }(<45 \mathrm{~g} / \mathrm{l}) \text {. }\end{array}$} \\
\hline
\end{tabular}


Table 4

FIP score before and after treatment in the remission group

\begin{tabular}{|c|c|c|c|c|c|c|c|c|}
\hline & Cat & Clinical signs & $\begin{array}{l}\text { FCoV } \\
\text { antibody } \\
\text { titre }\end{array}$ & Anaemia & Lymphopenia & $\begin{array}{l}\text { Hypergamma- } \\
\text { globulinaemia }\end{array}$ & Score & Score \\
\hline 1 & Yrael & Effusion & $\begin{array}{l}>1280 \\
/>1280\end{array}$ & NA & NA & NA & 2 & 2 \\
\hline 2 & $\begin{array}{l}\text { Charlie } \\
\text { Chaplin }\end{array}$ & $\begin{array}{l}\text { Neurological } \\
\text { signs. } \\
\text { Toxoplasmosis } \\
\text { co-infection. }\end{array}$ & $\begin{array}{l}>1280 \\
/>1280\end{array}$ & $\mathrm{NA} / \mathbb{\Delta}$ & 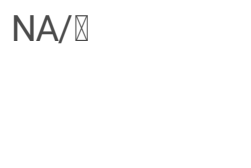 & $\nabla / \otimes$ & \multicolumn{2}{|c|}{$\begin{array}{l}\text { insufficient } \\
\text { data }\end{array}$} \\
\hline 3 & Smokey & $\begin{array}{l}\text { Failure to gain } \\
\text { weight and } \\
\text { enlarged MLN }\end{array}$ & $\begin{array}{l}>1280 \\
/>1280\end{array}$ & $\nabla / \nabla$ & $\nabla / \mathbb{Q}$ & 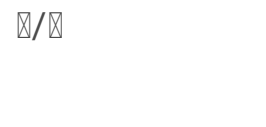 & 5 & 5 \\
\hline 4 & Rowley & $\begin{array}{l}\text { Ascites, then } \\
\text { uveitis, then } \\
\text { haemolytic } \\
\text { anaemia, } \\
\text { euthanasia }\end{array}$ & $\begin{array}{l}>1280 \\
/ 640\end{array}$ & $\nabla / \otimes$ & 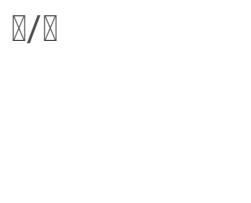 & $\nabla / \otimes$ & 4 & 3 \\
\hline 5 & Claude & $\begin{array}{l}\text { Pleural effusion, } \\
\text { deteriorated and } \\
\text { ascites appeared }\end{array}$ & NA & NA & NA & NA & \multicolumn{2}{|c|}{$\begin{array}{l}\text { insufficient } \\
\text { data }\end{array}$} \\
\hline 6 & Alfie & $\begin{array}{l}\text { Vomiting but } \\
\text { bright initially, } \\
\text { chronic diarrhoea, } \\
\text { enlarged MLN }\end{array}$ & $\begin{array}{l}>1280 \\
/ 1280\end{array}$ & NA & NA & NA & \multicolumn{2}{|c|}{$\begin{array}{l}\text { insufficient } \\
\text { data }\end{array}$} \\
\hline 7 & Holly & $\begin{array}{l}\text { Persistent } \\
\text { pyrexia, weight } \\
\text { loss then ascites }\end{array}$ & $\begin{array}{l}>1280 \\
/ 1280\end{array}$ & $\nabla / \nabla$ & 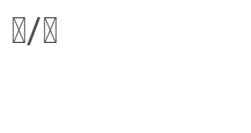 & 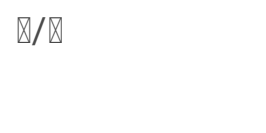 & 3 & 2 \\
\hline 8 & Bugsy & $\begin{array}{l}\text { Pyrexia, poor } \\
\text { body condition, } \\
\text { variable appetite }\end{array}$ & $\begin{array}{l}>1280 \\
/>1280\end{array}$ & $\nabla / \nabla$ & $\nabla / \nabla$ & 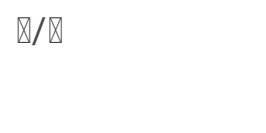 & 2 & 5 \\
\hline 9 & Daisy & $\begin{array}{l}\text { Weight loss, } \\
\text { intestinal } \\
\text { granuloma, } \\
\text { raised MLN. } \\
\text { Intestinal } \\
\text { granuloma } \\
\text { resolved with } \\
\text { treatment, but cat } \\
\text { still died }\end{array}$ & $\begin{array}{l}>1280 \\
/>1280\end{array}$ & $\nabla / \mathbb{\nabla}$ & $\nabla / \nabla$ & 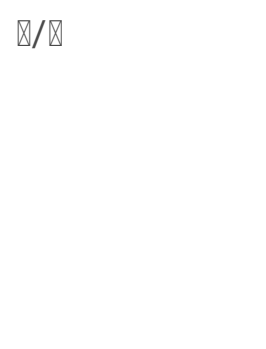 & 5 & 5 \\
\hline 10 & Levi & Ascites & $\begin{array}{l}>1280 \\
\text { /not } \\
\text { repeated }\end{array}$ & $\begin{array}{l}\bigotimes / \\
\bigotimes(\text { improved } \\
\text { though) }\end{array}$ & $\nabla / \otimes$ & 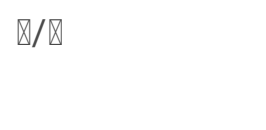 & 5 & 4 \\
\hline
\end{tabular}

NA: not available $邓$ present $\varangle$ - absent > - over

This table shows the clinical signs and scores before treatment and at death or last sampling, showing that treatment had not greatly improved the cat's score, and in some cases it worsened. First and latest FCoV antibody titres, presence or absence of anaemia, lymphopenia and hyperglobulinaemia are shown in the 4th, 5th, 6th and 7th columns respectively. In the last row, the totals for how many cats were anaemic, lymphopenic and hyperglobulinaemic at the start and end are shown, indicating that these clinical parameters deteriorated in this group of cats (although globulin levels reduced in four cats). 


\begin{tabular}{|c|c|c|c|c|c|c|c|c|}
\hline & Cat & Clinical signs & $\begin{array}{l}\text { FCoV } \\
\text { antibody } \\
\text { titre }\end{array}$ & Anaemia & Lymphopenia & $\begin{array}{l}\text { Hypergamma- } \\
\text { globulinaemia }\end{array}$ & Score & Score \\
\hline 11 & Roxanne & $\begin{array}{l}\text { Chronic } \\
\text { gingivostomatitis, } \\
\text { poor condition, } \\
\text { poor appetite, } \\
\text { jaundice which } \\
\text { resolved, but she } \\
\text { suddenly } \\
\text { developed ataxia } \\
\text { and was } \\
\text { euthanased }\end{array}$ & $\begin{array}{l}>1280 \\
/ 320\end{array}$ & 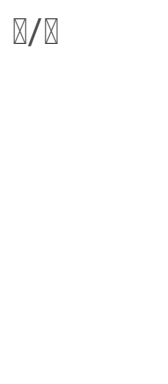 & 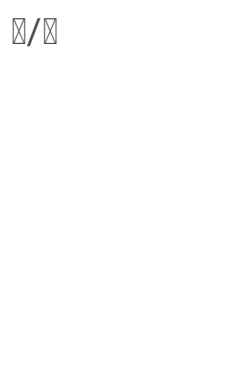 & 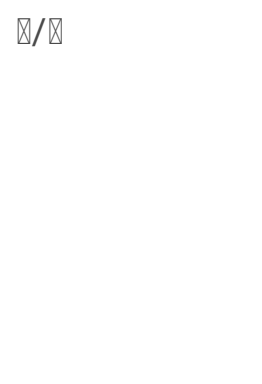 & 5 & 4 \\
\hline 12 & Pip & $\begin{array}{l}\text { Biopsied } 2004 . \\
\text { Feb 2005: } \\
\text { enlarged MLN, } \\
\text { weight loss, } \\
\text { anaemia, } \\
\text { reported well } 2 \text { m } \\
\text { later: remission } \\
\text { until } 10 \mathrm{~m} \text { then re- } \\
\text { presented } \\
\text { anaemic and } \\
\text { lymphopenic. }\end{array}$ & $\begin{array}{l}>1280 \\
/>1280\end{array}$ & 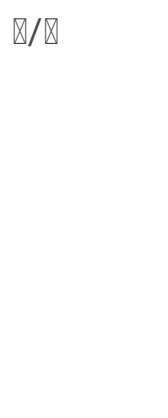 & low / $/$ & 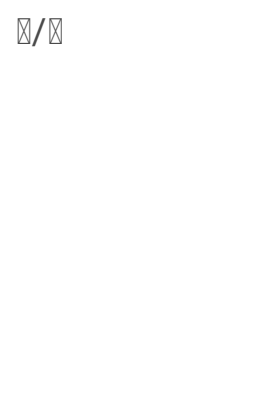 & 5 & 5 \\
\hline 13 & Pharaoh & $\begin{array}{l}\text { Weight loss, gut } \\
\text { biopsy showed } \\
\text { inflammation. }\end{array}$ & $\begin{array}{l}640 \\
/>1280\end{array}$ & $\nabla / \otimes$ & $\nabla / \otimes$ & $\nabla / \otimes$ & 3 & 5 \\
\hline 14 & Maximus & $\begin{array}{l}\text { Chronic } \\
\text { diarrhoea, poor } \\
\text { appetite. } \\
\text { Collapsed and } \\
\text { was euthanased. }\end{array}$ & $\begin{array}{l}>1280 \\
/>1280\end{array}$ & $\nabla / \nabla$ & $\nabla / \otimes$ & $\nabla / \nabla$ & 4 & 4 \\
\hline 15 & Ragamuffin & $\begin{array}{l}\text { Weight loss. MLN } \\
\text { enlarged, } \\
\text { diarrhoea, always } \\
\text { shed low } \\
\text { amounts of virus } \\
\text { in faeces, chronic } \\
\text { anaemia }\end{array}$ & $\begin{array}{l}>1280 \\
/>1280\end{array}$ & $\nabla / \otimes$ & $\begin{array}{l}\bigotimes / \\
\bigotimes(\text { sometimes } \\
\text { low normal) }\end{array}$ & $\nabla / \nabla$ & 4 & 5 \\
\hline 16 & Tinkerbell & $\begin{array}{l}\text { Underweight, } \\
\text { chronic poor } \\
\text { appetite. }\end{array}$ & $\begin{array}{l}>1280 \\
/ 1280\end{array}$ & $\nabla / \nabla$ & $\nabla / \nabla$ & 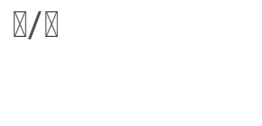 & 4 & 3 \\
\hline & Total & & & $\begin{array}{l}9 \text { of } 12 / \\
11 \text { of } 13\end{array}$ & $\begin{array}{l}6 \text { of } 11 / 11 \\
\text { of } 13\end{array}$ & $\begin{array}{l}11 \text { of } 13 / 9 \\
\text { of } 13\end{array}$ & & \\
\hline \multicolumn{9}{|c|}{ NA: not available $\bowtie$ present $\varangle$ - absent > - over } \\
\hline \multicolumn{9}{|c|}{$\begin{array}{l}\text { This table shows the clinical signs and scores before treatment and at death or last sampling, showing that treatment } \\
\text { had not greatly improved the cat's score, and in some cases it worsened. First and latest FCoV antibody titres, presence } \\
\text { or absence of anaemia, lymphopenia and hyperglobulinaemia are shown in the 4th, 5th, 6th and 7th columns } \\
\text { respectively. In the last row, the totals for how many cats were anaemic, lymphopenic and hyperglobulinaemic at the } \\
\text { start and end are shown, indicating that these clinical parameters deteriorated in this group of cats (although globulin } \\
\text { levels reduced in four cats). }\end{array}$} \\
\hline
\end{tabular}

Treatment. Treatment choice was at the discretion of the cat's attending veterinary surgeon or, more recently, the cat's guardian, with one exception: previously prednisolone was considered an integral part of FIP treatment ${ }^{32}$ but systemic corticosteroids were subsequently found to decrease survival time in cats that were being treated concurrently with polyprenyl immunostimulant (PI, VetImmune, Sass \& Sass, USA) ${ }^{16}$. Consequently, from 2017 one author (DDA) 
recommended stopping (or not commencing) the use of corticosteroids and to use meloxicam instead, (after a suitable wash out period and provided blood pressure and kidney function were normal).

Treatment protocols were variable, and are described to the best of our ability for each cat in Tables 1 and 2 . They most commonly included recombinant feline interferon omega ( $r F e l F N-\omega$, Virbac, France) by subcutaneous injection (for effusive FIP) or diluted and administered per os daily (for non-effusive FIP) ( $n=20$ recovered and 13 remission cats). Oral rFelFN- $\omega$ was also used as follow up to other therapies. Twelve cats were treated with a $5 \%$ oral adenosine nucleoside analogue (Mutian Xraphconn, Nantong Mutian Biotechnology Co. Ltd. China) ${ }^{33}$.

Supportive treatment included meloxicam per os daily; vitamin B12 (cobalamin) either as weekly injections or daily pills (Cobalaplex, Protexin Veterinary, UK); and Pro-kolin enterogenic probiotics (Protexin Veterinary, UK). Clients whose cats were treated with adenosine nucleoside analogue drugs were recommended to supplement with S-adenosyl-L-methionine (SAMe, Denamarin, Nutramax Laboratories, Inc, USA) for liver support.

Outcomes: death, recovery or remission. There are three possible outcomes following the diagnosis of FIP and treatment: death due to FIP-related causes; total recovery; or remission, defined as an intermediate stage between cure and death, carrying the spectre of relapse. This latter state is a source of considerable stress for cat guardians, so that it would bring considerable reassurance to people to know that their cats are cured of FIP, rather than being in remission. Death due to a non-FIP reason occurred in two cases (Edward and Bea) who were lost to follow-up due to a road accident and cancer.

\section{Case 1}

(Basil 1) set the benchmark for defining recovery from FIP against which other cases were compared. This cat was treated and was followed up for eleven years without relapsing. The criteria for declaring a cat recovered from FIP included the following:

- The cat returned to clinical normality, specifically resolution of the clinical signs of FIP.

- Globulin levels reduced to normal ( $\leq 45 \mathrm{~g} / \mathrm{l}$ ) or at least significantly reduced (often by over $15 \mathrm{~g} / \mathrm{l}$ as shown in Table 3).

- Resolution of lymphopenia, where present, (normal defined as greater than $1.5 \times 10^{9} / \mathrm{I}$ ).

- Haematocrit level increased to normal (defined as $30 \%$ or over), with reversal of non-regenerative anaemia, where present.

- At least three-fold reduction in FCoV antibody titre.

- AGP levels returned to normal levels $(\leq 500 \mu \mathrm{g} / \mathrm{ml})^{12}$.

Clinical scores are shown in Tables 3 and 4. Since the purpose of this study was to assess whether or not AGP reduction was an indicator of clinical recovery, AGP was not included in the cat's score for definition of a particular case as recovered or only in remission.

Cats were deemed to be in remission if only some of the criteria listed above were met: for example, cat 15 (Ragamuffin) survived over 5 years but she was never clinically well during that time, requiring continuous treatment, and unfortunately she was lost to follow up.

AGP. AGP was measured by enzyme-linked immunosorbent assay (ELISA) (Avacta Animal Health, Wetherby, Yorkshire, UK) at the University of Glasgow Veterinary Diagnostic Services (VDS) in all cases except seven: the samples of Basil 1; Amy; Brook; Bugsy; Daisy; Roxanne and Pip were measured by radial immunodiffusion as previously described ${ }^{12}$. 
The maximum value given on the $y$ axis of Figures 1 and 2 was set at $5000 \mu \mathrm{g} / \mathrm{ml}$ in order to maintain legibility of the graphs. The minimum ELISA cut-off was $300 \mu \mathrm{g} / \mathrm{ml}$ and results reported as below 300 were plotted as 300 .

Statistics. Due to small sample size, the Fisher's exact test was used with significance set at $<0.05$.

Ethical approval. All applicable international, national and institutional guidelines for the care of animals were followed. All procedures performed in studies involving animals were in accordance with the ethical standards of the governing bodies of the veterinary surgeons of the countries in which the studies were conducted.

The reporting in this manuscript follows the recommendations in the ARRIVE guidelines.

Informed consent was obtained from the cat guardians for publication of their pet's data.

\section{Results}

The laboratory records of effusive $(n=17)$ and non-effusive $(n=25)$ FIP cases were reviewed.

Recovery, remission, and death. As shown in Tables 1 and 2, 26 cats recovered from FIP, with follow up periods of up to 11 years. Of the recovered cases, 11/26 (42\%) had effusive FIP and 14 /26 (54\%) were non-effusive. One cat (Nelson) with non-effusive FIP developed an effusion following biopsy; another cat (Boris) had non-effusive FIP, initially thought to be effusive, but later established to be a cardiogenic effusion (FCoV RT-PCR on his effusion was negative). Two of the noneffusive FIP cases were the colonic presentation.

One cat (Kitten 2) in the recovered group experienced a relapse: details are given below.

Sixteen cats experienced remissions of 1.5 to over 60 months. Median remission was 4.5 months (not including the outlier of Ragamuffin who was lost to follow up after 5 years because her result would skew the median). Twelve cats died or were euthanased and 4 were lost to follow up.

Amongst the cats who experienced remission, four (25\%) had effusive FIP (one - Rowley- became non-effusive) and 12 (75\%) had non-effusive FIP (one - Holly - became effusive).

Twelve of 17 (71\%) effusive cases and 14/25 (56\%) non-effusive FIP cases recovered. Initial effusive or non-effusive form did not affect whether or not a cat fully recovered (Fisher's exact, $p=0.52$ ).

AGP levels. AGP levels of recovered cats and those who experienced remission are shown in Figures 1 and 2 respectively. AGP levels were elevated (i.e., above $500 \mu \mathrm{g} / \mathrm{ml}$ ) in all FIP cases except in the cases of Wish and Mike, where the first AGP test was taken after treatment had started, so the pre-treatment AGP results were unknown.

The time for AGP levels to return to normal in recovered cats varied from a minimum of less than 13, 20 and 22 days (Wish, Lyra and Basil 1) to over 16 months from onset of treatment, probably depending on the treatment being used (Table 1). The AGP levels of one recovered cat (Chynah) did not return to normal and with the benefit of hindsight, this cat should perhaps be in the remission group although her veterinary surgeon reported that she was clinically recovered: unfortunately, she was lost to follow up.

Two of the cats (Holly and Daisy) who did not recover had AGP levels which reduced to under $500 \mu \mathrm{g} / \mathrm{ml}$ on one occasion each and AGP levels reduced to under $1000 \mu \mathrm{g} / \mathrm{ml}$ in two more cats. Therefore, it is important that two consecutive normal AGP results at least one week apart be obtained to ensure that recovery from FIP has occurred.

Of particular interest was the AGP level of Kitten 2 (Figure 3) who was diagnosed with effusive FIP in October 2019. Her guardian stopped the oral adenosine nucleoside analogue (Mutian X) treatment on January $5^{\text {th }} 2020$ following an AGP 
result of $709 \mu \mathrm{g} / \mathrm{ml}$. This young cat presented with an FIP relapse in February 2020 manifesting as hyperesthesia of the tail, which progressed to ataxia, then seizures. A second course was administered, this time at a double dose (one Mutian $X 200$ per kg) which is a level that enables sufficient anti-viral drug to cross the blood brain barrier (Tony Xue, personal communication). The cat improved within 24 hours and the second course of Mutian $X$ was continued until AGP reached below $500 \mu \mathrm{g} / \mathrm{ml}$ : she is alive and well 17 months later. After this event, FIP cat guardians were recommended to give a double dose of oral (not injectable) Mutian for 7-10 days to clear virus from the brain to prevent neurological relapse.

Haematocrit. A lower cut-off of $30 \%$ was our definition of normal below which a cat was said to be anaemic. This is the value determined by University of Glasgow Veterinary Diagnostic Services Laboratory.

Haematocrit was available for 25 of 26 recovered cats and 13 of 16 cats who went into remission. (Tables 3 and 4 ). One recovered cat (Chynah) and 3 remission cats (Yrael, Claude and Alfie) had no haematology results and in five recovered cats (Dante, Molly, Nelson, Edward, Kitten 2) records were not available until after treatment began - too late to determine whether or not they had been anaemic because by that time they were not anaemic. In two cats Hct was only marginally below normal (Tabitha $29.4 \%$ and Chester: $28.9 \%$ ) on one occasion only, therefore they were not seriously anaemic and were discounted from analyses. Basil 2's anaemia was complicated by concurrent haemoplasmosis infection (Figure 4). This left usable haematocrit records for 15 recovered cats and 13 cats who went into remission: 6 of the 15 recovered cats had effusive FIP and 9 had non-effusive FIP; 2 remission cats had effusive FIP and 11 remission cats had non-effusive FIP.

\section{Table 5. Summary of anaemia}

\begin{tabular}{|llll|}
\hline & Anaemic & Not anaemic & Total \\
\hline Recovered effusive & 6 & 1 & 7 \\
\hline Recovered non-effusive & 5 & 5 & 10 \\
\hline Remission effusive (but 1 became non-effusive) & 2 & 0 & 2 \\
\hline Remission non-effusive (but one became effusive) & 10 & 1 & 11 \\
\hline
\end{tabular}

Eight of 9 (89\%) cats with effusive FIP and 15 of 21 (71\%) cats with non-effusive FIP were anaemic (Tables 3, 4 and 5). Whether FIP was effusive or non-effusive made no statistical difference to the likelihood of the cat being anaemic $(p=$ $0.70)$.

Eleven of $17(65 \%)$ cured and 12 of $13(92 \%)$ cats in remission were anaemic: the presence of anaemia did not affect the cat's chances of recovery $(p=0.10)$.

Two remission cats became anaemic after FIP diagnosis, and one cat's anaemia resolved. Anaemia resolved in all of the cats who recovered, but Basil 2 required a one-month course of doxycycline for concurrent haemotropic mycoplasma infection for his anaemia to resolve. The haematocrit of Basil 2 is shown in Figure 4: it illustrates the typical wave pattern due to cyclical parasitemia with a periodicity of 7-10 days, resolving when doxycycline was begun.

While the average time for resolution of purely FIP related anaemia was 30.4 days in 10 cats (range 12 to 57 days: Table 3 ), from initiation of treatment, the average time for AGP to return to WNL was 45 days (range 20-117 days, not counting the two cats for whom there was no pre-treatment AGP). The outlier cat, Mike, was not counted in this analysis because there was no initial AGP for comparison: his anaemia resolved 154 days after treatment began.

Lymphopenia. A lymphocyte count of $1.5 \times 10^{9} /$ I was considered the lowest level at which lymphocyte count could be considered normal: this is the cut-off set by the VDS laboratory. Sixteen of 37 (43\%) cats with FIP were lymphopenic on 
the first available lymphocyte count closest to FIP diagnosis. The prevalence of lymphopenia was $38.5 \%(5 / 13)$ in effusive cases and $45.8 \%(11 / 24)$ in non-effusive cases.

Sequential lymphocyte counts were available for 25 of 26 recovered cats and 13 of 16 remission cats, but the first count was two months after treatment began for one recovered cat (Edward). Although the median lymphocyte count was slightly higher for recovered cats $\left(1.833\right.$ vs $\left.1.366 \times 10^{9} / \mathrm{l}\right)$, the difference was not significantly different between the recovered or remission groups $(p=0.47)$.

It appeared that fewer recovered cats (9/24: 37\%) than remission cats (7/13: $54 \%)$ were lymphopenic, but the difference was not statistically different $(p=0.5)$. Four recovered cats had very low lymphocyte counts but within the normal range (1.5 to $7.0 \times 10^{9} /$ I): one cat (Buddie) was lymphopenic on 2 of 7 samples and another cat (Molly) had low lymphocyte counts (below $2 \times 10^{9} /$, but above $1.5 \times 10^{9} /$ I which is considered the lower cut-off).

Lymphopenia resolved in all recovered cats. In the remission group, three cats became lymphopenic and the status of the other cats remained the same (Table 4).

Hyperglobulinaemia. Globulin levels were available for all the 26 recovered cats and for 13/16 remission cats, but there was no pre-treatment sample for one of the recovered cats and by the time he was tested his globulin levels were normal. Twenty-three of the remaining 25 recovered cats were hyperglobulinemic: globulin levels reduced in all 23 cats, to WNL in 16 cats, but were still elevated in 7 cats.

Eleven of 13 remission cats were hyperglobulinemic and globulin levels reduced in 4 cats.

AGP was a more accurate prognostic indicator than globulin reduction because globulins were slower to reduce than AGP level in recovered cats (7 of 23 cats still had elevated globulin levels once recovered) and they reduced in 4 of 12 remission cases.

FCoV antibody titre. As shown in Tables 3 and 4, all of the cats had very high FCoV antibody titres, being on or above the upper cut off point for the laboratory to which samples had been sent. Two exceptions were Nelson, who recovered, and the remission cat Pharaoh, whose FCoV antibody titre was only moderately high at 640 at time of diagnosis, but subsequently became very high. FCoV antibody titres remained high in almost all recovered cats for a very long period, even years (Table 3): the earliest that a significant reduction in FCoV antibody titre was seen was at 4 months postdiagnosis, and one cat became seronegative at 8 months.

FCoV antibody titres in the remission group did not decrease, with the exception of Rowley, whose titre decreased from $>1280$ to 640 , likely due to immunosuppressive amounts of prednisolone with which he was being treated.

\section{Discussion}

Recovery implies that the disease is finished once and for all, whereas remission is defined as the reduction or diminution of clinical signs, with the implication that the disease could reappear. The state of remission in our series usually culminated in death, mainly by euthanasia due to a prolonged cachexic state, or sometimes acutely, presenting as acute haemolytic anaemia, collapse, or neurological signs. While parameters and a score system for predicting imminent death have been well documented ${ }^{10}$, no such system has been previously defined to differentiate recovery from remission. We present the first documentation of sequential AGP testing of cats being treated for FIP and present evidence that a consistent reduction in AGP to normal levels is the most useful marker for differentiating recovery from remission, and is a clear indicator that it is safe to stop administering adenosine nucleoside analogue anti-viral drugs. 
Raised AGP was shown to be $100 \%$ sensitive in FIP diagnosis ${ }^{12,13}$ but is not specific: it rises in both transient FCoV infection $^{34}$ and in other infections (e.g. bacterial peritonitis or pleurisy; Mycoplasma haemofelis infection ${ }^{35}$ ). However, the sensitivity of AGP measurement in cats with FIP only in the brain has not been established: Rissi et al, $2018^{36}$ reported that five of 22 cats with neurological FIP had no FIP lesions in other organs, and it is possible that in those cats AGP measurement would not have been raised. Raised AGP was more sensitive than histopathology for diagnosing FIP in challenging cases ${ }^{13}$ and this was also true in our series, where histopathology-especially of the MLN-was frequently non-specific and reported as simply pyogranulomatous inflammation.

AGP was the most useful parameter for assessing recovery from FIP because it was raised in all our cases, whereas anaemia, lymphopenia and hyperglobulinaemia were not consistently found. Anaemia resolved more quickly than did AGP in five of six of the nine anaemic cats in which the time to return to normal was not the same for both AGP and Hct (in three cats the identical intervals presumably simply reflected the intervals between blood samplings). Consequently, where AGP measurement is not possible and if the cat is anaemic purely due to FIP (e.g. uncomplicated by concurrent haemoplasmosis), then return of Hct to over $30 \%$ appears to be a good indicator of recovery, although our numbers were small. However, the anaemia of one of the cats in the remission group also resolved, so resolution of anaemia alone is not a guarantee of recovery.

The exact interval from FIP diagnosis to normal AGP could not be accurately determined because it depended on the frequency of the cat being blood tested, which was at the discretion of the cat's guardian: we were only able to determine that it was less than however many days from the start of treatment to the first AGP test WNL. However, the intervals appeared to be noticeably shorter after 2019, when adenosine nucleoside analogue drugs were introduced ${ }^{19}$. The earliest that AGP returned to normal was around two to three weeks from the start of treatment.

The prevalence of lymphopenia in this study (43\%) was similar to that of other studies: $49.5 \%$ of 184 cats with FIP ${ }^{9}$; $50 \%$ of 106 cats $^{11}$. and $64 \%$ of 45 cats $^{10}$. In the study of Riemer et al $2016^{9}$, lymphopenia was observed significantly more often in 139 cats with effusion and was documented in only $26.8 \%$ of 41 cats without effusion. Our results differed: $38.5 \%$ of effusive cases were lymphopenic compared with $48.8 \%$ of non-effusive cases, but our cohort of cats was much smaller than that of Riemer et al, $2016^{9}$.

In the study of Tsai et al (2011) ${ }^{10}$ the prevalence of lymphopenia was $64 \%$ at initial presentation and increased to $91.7 \%$ zero to three days before death. In our study, lymphopenia reversed in the lymphopenic cats who recovered, and two cats in the remission group became lymphopenic. Accordingly, the development of lymphopenia in a cat with FIP who had not previously been lymphopenic is a poor prognostic sign. Our study could not assess the utility of documenting reversal of lymphopenia as a marker for recovery from FIP, because unfortunately during most of this study prednisolone was given to treat FIP, which would have interfered with (i.e. decreased or suppressed) the lymphocyte count. Further studies will be required to assess the utility of lymphopenia reversal as an indicator of FIP recovery, although given that around half of cats with FIP are not lymphopenic, it will clearly be less useful than AGP, which is consistently elevated in cats with FIP.

The definitive marker for recovery from FCoV infection is the reduction of FCoV antibody titre to undetectable levels, signifying that viral antigen no longer remains in the body to stimulate an immune response; but this takes many months to achieve, consequently it is not a useful marker to determine when to stop treatment. In FIP recovered cats, significant reduction of FCoV antibody titre takes so long to achieve (usually over one year) that it would be inadvisable to sustain anti-viral treatment long term due to the risk of toxic adverse effects to the kidneys and liver. In contrast, antibody levels in FCoV infected cats without FIP decline within a few months of virus being eliminated from the intestine and faeces (data not shown).

We observed two relapsed cases who presented with a painful tail: Kitten 2, described in this paper, and another cat for whom we had no AGP results so was not documented here. The occurrence of idiopathic painful tail syndrome has been 
reported previously ${ }^{37}$ and André et al, $2019^{38}$, described a neurological FIP case which began as plegia of the tail. FIP is the major cause of hydrocephalus in young cats $36,39,40$. Cerebrospinal fluid (CSF) drains from the thecal sac at the level of the second sacral vertebra and it is likely that build up of CSF in this area pressed on the nerves of the cauda equina, causing an initial presentation of sensitivity and pain in the tail. As CSF built up in the ventricular system of the central nervous system the two cats progressed to show hind limb ataxia, then seizures. Fortunately, prompt administration of antiviral pills to Kitten 2 saved her life; the other cat was treated with an injectable nucleoside analogue (Mutian II), but died. Two other cases (outwith this study) treated with injectable nucleoside analogues have presented with neurological relapses, but recovered after a course of double dose pills: it seems counterintuitive for an oral formulation to cross the blood brain barrier more effectively than the injectable formulations, but that is our experience. Following the Kitten 2 relapse, clients were recommended to give a 7-10-day double dose of Mutian X to clear the brain of virus.

It might be wondered why there was only one relapse amongst the 17 cats (6\%) which were treated with nucleoside analogue drugs when in the first study where nucleoside analogues were used there were eight relapses in 26 cats (31\%) ${ }^{19}$. We believe that one of the most important reasons was that 12 cats were treated with an oral adenosine nucleoside analogue, which accesses the site of viral replication in the intestine and stopped virus shedding in faeces in all cases. In contrast, one cat (Mike) treated with injectable GS-441524 continued shedding virus even two years after diagnosis. A similar problem has been encountered in humans treated with Remdesivir, the GS-441524 pro-drug (with or without convalescent plasma) where prolonged virus shedding was reported in 13 patients ${ }^{41}$. It may be that this anti-viral fails to adequately reach every organ, allowing pockets of virus to survive and recrudesce ${ }^{42}$. One patient that was not in our series (because AGP was not monitored) was presented with relapse of FIP pleural effusion. Initially that cat had been treated with ten days of Mutian II injections which may have failed to properly clear the virus from his lungs: this was seen in an immunocompromised human COVID19 patient treated with Remdesivir ${ }^{42}$.

Other factors which contributed to a successful outcome included being able to differentiate accurately between recovery and remission using AGP levels, therefore knowing when it was safe to discontinue the antiviral drug; secondly anti-viral treatment was followed up with long term oral feline interferon which has anti-viral and immunomodulatory activity: and thirdly, in-contact cats were tested for FCoV shedding and if positive, they were treated to stop virus shedding ${ }^{33}$ thus re- $^{-}$ infection was prevented (presumably re-infection could appear as a relapse). The approach of using anti-virals in subclinically infected cats has a parallel in SARS-CoV2 infection where two doses of prophylactic ivermectin reduced the risk of COVID-19 infection by $83 \%$ in the following month in health care workers in India ${ }^{43}$.

It should always be considered that the appearance of relapse or failure to respond to treatment may, in reality, be due to secondary conditions, for example the anaemia of Basil 2 was due to concurrent haemoplasmosis but initially appeared to be a lack of response to FIP treatment.

It is likely that immunosuppression due to FIP-induced lymphopenia leads to secondary infections in FIP. Thus, in addition to infectious anaemia, we have observed toxoplasmosis and clinical signs attributable to recrudescent latent feline herpesvirus infection, such as epiphora and sneezing. One relatively frequent curious condition that occurred was trichobezoar which is not explicable by immunosuppression. In most cases this was easily dealt with using a psylliumcontaining dry cat food (Royal Canin Aging 12+) but in one cat, who was not included in this series due to lack of AGP results, surgery was required to remove a large furball.

A failing of our study was the inability to compare weight gain or loss in the two groups. In a previous study, weight gain was an important measurement of treatment success ${ }^{19}$. Unfortunately weight records were only available for the most recent recovered cases and none in the remission group.

This study might be criticised for a lack of histopathological confirmation of FIP in many of the cases. Since the cats were field cases, it would not have been ethical to ask for biopsies to be performed for the sake of this study, and most

Page 24/31 
guardians did not elect to have histopathological confirmation of diagnosis in those cats who died. Many of the cats had positive FCoV RT-PCR tests of effusion or MLN FNA and such evidence has been accepted as diagnostic of FIP in other therapeutic studies ${ }^{18,19}$. Demonstration of replicating FCoV by messenger RNA RT-PCR ${ }^{26}$ in peripheral blood mononuclear cells (pbmc) was considered diagnostic of FIP, but it was borne in mind that FCoV mRNA had been detected in $5 \%$ of cats without FIP 26,27 and during this study, we found that the primers cross-reacted with human DNA, giving false positive results on some samples (therefore positive pbmc mRNA results were confirmed by a 3' UTR RT-PCR).

There remained some cats who were diagnosed only by circumstantial evidence, but in all cases elevated FCoV antibody titres and AGP levels were documented, and response to anti-coronavirus drugs was further corroboration of a correct FIP diagnosis.

\section{Conclusion}

As new FIP treatments become available we have shown that AGP measurement will be a useful parameter to assess their efficacy. Increasing AGP can indicate that a cat's condition is deteriorating but a return to $500 \mu \mathrm{g} / \mathrm{l}$ or less sustained for at least one week indicated recovery from FIP and differentiated recovered cats from those in remission. It is inadvisable to reduce the dosage or frequency of treatment, even if clinical signs appear to have resolved, until AGP levels are once again normal.

\section{Declarations}

\section{Acknowledgements}

We are very grateful to the guardians of these cats for consenting for the publication of their cases, amongst whom were Helen Alford, James Arnold, Kirsty Bedford, Nancy Court, Diana Donciu, Sarah Edwards, Mrs Evans, Mrs Franklin, Jake Goldstein, Peter and Rochelle Harisis, Bianca Houlgrave, Eric and Carrie Johnson, Mrs Lake, Karen Major, Claire NellerClements, Tanya Osborne, Mr Slocombe, Sarah Stoddart, Sarah Stokes.

The authors sincerely thank the following veterinary surgeons for providing information and samples: David Abratt, Andrew Armitage, Roger Bralow, Colin Dick, Rebecca Gibb, Stuart Hills, Hannah Johnston, Colin Lyons, Carol Mason, Andy Nelson, Graham Pursey, Fiona Thomson, Jason Way.

We thank Oswald Jarrett, Flora Bellini and Ben Crowe for scientific editing. We are grateful to the technicians, office and veterinary staff of Glasgow University Veterinary Diagnostic Services Laboratory, with special thanks to Leigh Marshall for AGP and FCoV antibody testing, and Dawn Dunbar and Mike McDonald for FCoV RT-PCR testing.

We are deeply indebted to the Angelica Trust donors to the www.catvirus.com website for support of D.D.A. during the writing of this paper and for covering publication fees.

\section{Author contributions}

Study conception and design: D.D.A. Data collection: D.D.A., C.S., C.A., P.B., J. C-R., C.F., M.F., C.G., K.M., J.M., E.P. and E.R. Data analysis: D.D.A and C.S. Manuscript writing: D.D.A., C.S. All authors reviewed the manuscript.

\section{Competing interests}

D.D.A. is a former employee of VDS. She received commissions from Virbac in 2004 and Royal Canin in 2021. She is currently a member of the European Advisory Board on Cat Diseases (ABCD), which is sponsored by Boehringer Ingelheim and Virbac: ABCD members receive no remuneration. No commercial company was involved at any stage in this study, or 
had any influence on the decision to publish the findings. None of the other authors declare any potential competing interests.

\section{Data availability}

All data generated or analysed during this study are included in this published article.

\section{References}

1. Herrewegh, A.A.P.M., Smeenk I., Horzinek M.C., Rottier P.J.M., de Groot R.J. Feline coronavirus type II strains 79-1683 and 79-1146 originate from a double recombination between feline coronavirus type I and canine coronavirus. J. Virol. 72, (5) 4508-4514. (1998).

2. Terada, Y., Matsui, N., Noguchi, K., Kuwata, R., Shimoda, H., Soma, T., Mochizuki, M., Maeda, K. Emergence of pathogenic coronaviruses in cats by homologous recombination between feline and canine coronaviruses. PLoS One. 9(9):e106534. (2014).

3. Kipar, A., May, H., Menger, S., Weber, M., Leukert mW., Reinacher, M. Morphologic features and development of granulomatous vasculitis in feline infectious peritonitis. Vet Pathol. 42(3),321-30. (2005).

4. Addie, D.D., Toth S, Murray G.D., Jarrett, O. The risk of feline infectious peritonitis in cats naturally infected with feline coronavirus. Am. J. Vet. Res. 56, 4; 429-434. (1995).

5. Takano, T. et al. A "possible" involvement of TNF-alpha in apoptosis induction in peripheral blood lymphocytes of cats with feline infectious peritonitis. Vet Microbiol. 119, 121-31. (2007).

6. Takano, T. et al. Neutrophil survival factors (TNF-alpha, GM-CSF, and G-CSF) produced by macrophages in cats infected with feline infectious peritonitis virus contribute to the pathogenesis of granulomatous lesions. Arch Virol. 154, 775-81. (2009).

7. Goitsuka, R. et al. IL-6 activity in feline infectious peritonitis. J. Immunol. 144, 2599-2603. (1990).

8. Haagmans, B.L., Egberink, H.F., Horzinek, M.C. 1Apoptosis and T-cell depletion during feline infectious peritonitis. J. Virol. 70, 8977-8983. (1996).

9. Riemer, F., Kuehner, K.A., Ritz, S., Sauter-Louis, C., Hartmann, K. Clinical and laboratory features of cats with feline infectious peritonitis - a retrospective study of 231 confirmed cases (2000-2010). J. Feline Med Surg. 18, 348-56. (2016).

10. Tsai, H.Y., Chueh, L.L., Lin, C.N., Su, B.L. Clinicopathological findings and disease staging of feline infectious peritonitis: 51 cases from 2003 to 2009 in Taiwan. J. Feline Med Surg. 13, 74-80. (2011).

11. Yin, Y. et al. A retrospective study of clinical and laboratory features and treatment on cats highly suspected of feline infectious peritonitis in Wuhan, China. Nature Sci Rep. 11, 5208. (2021).

12. Duthie, S., Eckersall, P.D., Addie, D.D., Lawrence, C.E., Jarrett,O. Value of a1-acid glycoprotein in the diagnosis of feline infectious peritonitis. Veterinary Record 141, 299-303. (1997).

13. Giori, L., Giordano, A., Giudice, C., Grieco, V., Paltrinieri, S. Performances of different diagnostic tests for feline infectious peritonitis in challenging clinical cases. J. Small Anim. Pract. 52, 152-157. (2011).

14. Hsiao, S.Y. et al. $a$-1-Acid Glycoprotein Concentration as an Outcome Predictor in Adult Patients with Sepsis. Biomed Res Int. 3174896. (2019).

15. Ishida, T., Shibanai, A., Tanaka, S., Uchida, K., Mochizuki, M. Use of recombinant feline interferon and glucocorticoid in the treatment of feline infectious peritonitis. J. Feline Med. Surg. 6, 107-109. (2004).

16. Legendre, A.M., Kuritz, T., Galyon, G., Baylor, V.M., Heidel, R.E. Polyprenyl Immunostimulant Treatment of Cats with Presumptive Non-Effusive Feline Infectious Peritonitis in a Field Study. Front Vet. Sci. 4, 7. (2017). 
17. Hugo, T.B. \& Heading, K.L. Prolonged survival of a cat diagnosed with feline infectious peritonitis by immunohistochemistry. Can. Vet. J. 56, 53-8. (2015).

18. Pedersen, N.C. et al. Efficacy of a 3C-like protease inhibitor in treating various forms of acquired feline infectious peritonitis. J. Feline Med. Surg. 20, 378-392. (2018).

19. Pedersen, N.C. et al. Efficacy and safety of the nucleoside analog GS-441524 for treatment of cats with naturally occurring feline infectious peritonitis. J. Feline Med. Surg. 21, 271-281. (2019).

20. Herrewegh, A.A.P.M. et al. Detection of feline coronavirus RNA in feces, tissue, and body fluids of naturally infected cats by reverse transcriptase PCR. J. Clin. Microbiol. 33, 684-689. (1995).

21. Gut, M., Leutenegger, C.M., Huder, J., Pedersen, N.C., Lutz, H. One-tube fluorogenic reverse transcription-polymerase chain reaction for the quantitation of feline coronaviruses. J. Virological Methods. 77, 37-46. (1999).

22. Doenges, S.J., Weber, K., Dorsch, R., Fux, R., Hartmann, K. Comparison of real-time reverse transcriptase polymerase chain reaction of peripheral blood mononuclear cells, serum and cell-free body cavity effusion for the diagnosis of feline infectious peritonitis. J. Feline Med. Surg. 19, 344-350. (2017).

23. Longstaff, L. et al. Feline coronavirus quantitative reverse transcriptase polymerase chain reaction on effusion samples in cats with and without feline infectious peritonitis. J. Feline Med. Surg. 19, 240-245. (2017).

24. Tasker, S. Diagnosis of feline infectious peritonitis: Update on evidence supporting available tests. J. Feline Med. Surg. 2018, 228-243. (2018).

25. Yen, S.-J. \& Chen, H-W. Feline Coronaviruses Identified in Feline Effusions in Suspected Cases of Feline Infectious Peritonitis. Microorganisms. 9, 1801. (2021).

26. Simons, F.A. et al. A mRNA PCR for the diagnosis of feline infectious peritonitis. J. Virol. Methods 124, 111-116. (2005).

27. Fish, E.J. et al. Cross-sectional quantitative RT-PCR study of feline coronavirus viremia and replication in peripheral blood of healthy shelter cats in Southern California. J. Feline Med. Surg. 20, 295-301. (2018).

28. Dunbar, D. et al. Diagnosis of non-effusive feline infectious peritonitis by reverse transcriptase quantitative polymerase chain reaction from mesenteric lymph node fine needle aspirates. J. Feline Med. Surg. 21, 910-921. (2019).

29. Chang, H.W., Egberink, H.F., Halpin, R., Spiro, D.J., Rottier, P.J. Spike protein fusion peptide and feline coronavirus virulence. Emerg. Infect. Dis. 18, 1089-1095. (2012).

30. Barker EN, Stranieri A, Helps CR, Porter EL, Davidson AD, Day MJ, Knowles T, Kipar A, Tasker S. Limitations of using feline coronavirus spike protein gene mutations to diagnose feline infectious peritonitis. Vet. Res. 48, 60. (2017).

31. Porter, E. et al. Amino acid changes in the spike protein of feline coronavirus correlate with systemic spread of virus from the intestine and not with feline infectious peritonitis. Vet. Res. 45, 49. (2014).

32. Hartmann, K., Ritz, S. Treatment of cats with feline infectious peritonitis. Vet. Immunol. Immunopathol. 123(1-2):1725. (2008).

33. Addie, D.D. et al. Oral Mutian® X stopped faecal feline coronavirus shedding by naturally infected cats. Res. Vet. Sci. 130, 222-229. (2020).

34. Giordano, A., Spagnolo, V., Colombo, A., Paltrinieri, S. Changes in some acute phase protein and immunoglobulin concentrations in cats affected by feline infectious peritonitis (FIP) or exposed to feline coronavirus infection. Vet. $J$. 167, 38-44. (2004).

35. Korman, R.M. et al. Acute phase response to Mycoplasma haemofelis and 'Candidatus Mycoplasma haemominutum' infection in FIV-infected and non-FIV-infected cats. Vet. J. 193, 433-8. (2012).

36. Rissi. D.R. A retrospective study of the neuropathology and diagnosis of naturally occurring feline infectious peritonitis. J. Vet. Diagn. Invest. 30, 392-399. (2018).

Page 27/31 
37. Amengual Batle, P., Rusbridge, C, Nuttall T, Heath S, Marioni-Henry. K. Feline hyperaesthesia syndrome with selftrauma to the tail: retrospective study of seven cases and proposal for an integrated multidisciplinary diagnostic approach. J. Feline Med. Surg. 21, 178-185. (2019).

38. André, N.M., Cossic, B., Davies, E., Miller, A.D., Whittaker, G.R. Distinct mutation in the feline coronavirus spike protein cleavage activation site in a cat with feline infectious peritonitis-associated meningoencephalomyelitis. JFMS Open Rep. 26;5(1):2055116919856103. (2019).

39. Penderis J. The Wobbly Cat. Diagnostic and therapeutic approach to generalised ataxia. J. Feline Med. Surg. 11: 349359. (2009).

40. Crawford, A.H. et al. Clinicopathologic Features and Magnetic Resonance Imaging Findings in 24 Cats With Histopathologically Confirmed Neurologic Feline Infectious Peritonitis. J. Vet Intern Med. 31(5):1477-1486. (2017).

41. Drancourt, M. et al. SARS-CoV-2 Persistent Viral Shedding in the Context of Hydroxychloroquine-Azithromycin Treatment. Viruses. 13(5):890. (2021).

42. Choi, B. et al. Persistence and Evolution of SARS-CoV-2 in an Immunocompromised Host. N Engl J Med. 383(23):2291-2293. (2020).

43. Behera, P. et al. Prophylactic Role of Ivermectin in Severe Acute Respiratory Syndrome Coronavirus 2 Infection Among Healthcare Workers. Cureus. 13(8): e16897. doi:10.7759/cureus.16897. (2021).

44. Jones, S., Novicoff, W., Nadeau, J., Evans, SW. Unlicensed GS-441524-Like Antiviral Therapy Can Be Effective for atHome Treatment of Feline Infectious Peritonitis. Animals 11, no. 8: 2257. (2021).

\section{Figures}

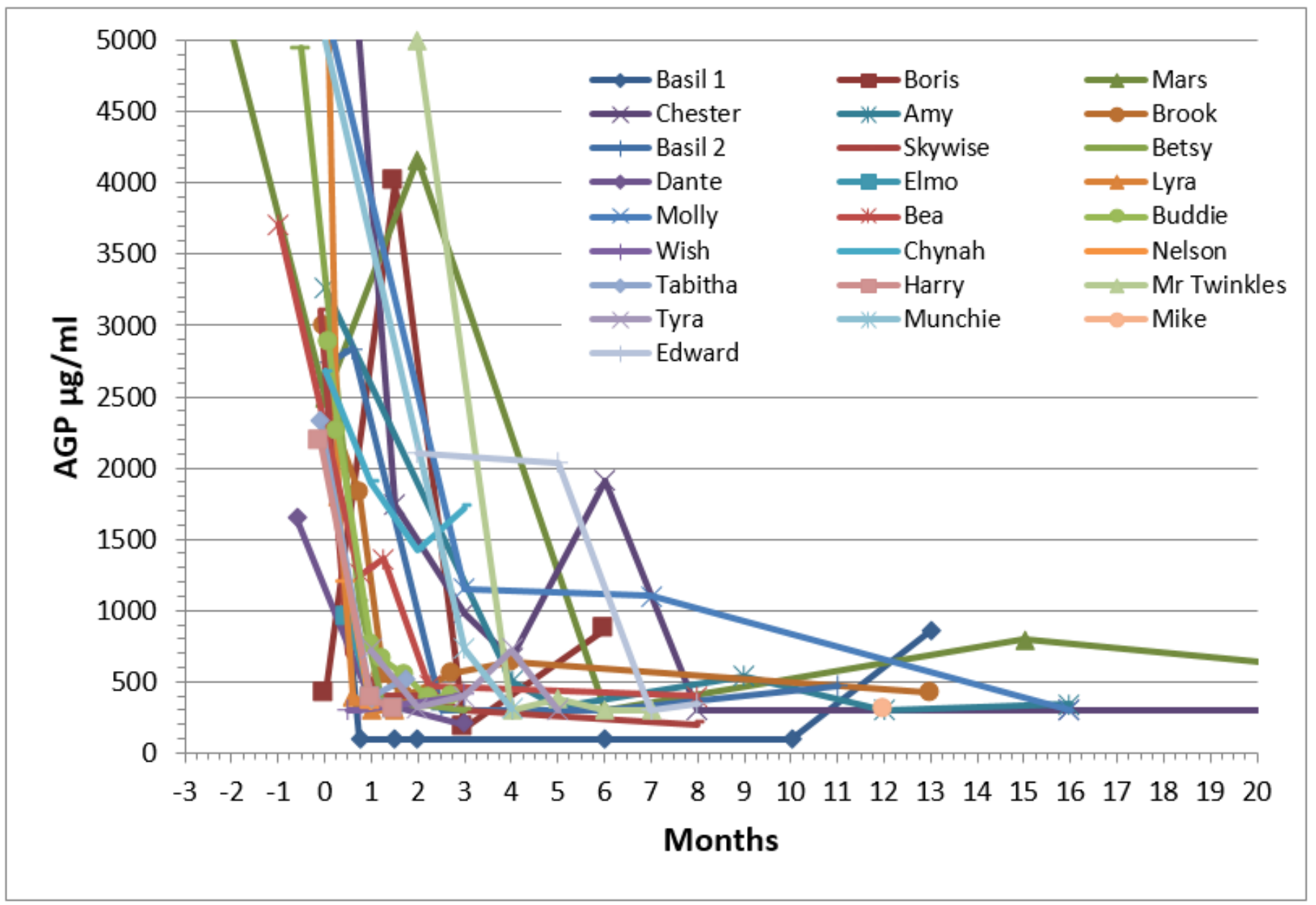




\section{Figure 1}

\section{Sequential AGP results of 25 cats who recovered from FIP}

This graph shows the time in months for AGP levels to return to normal, with Day 0 being the first day of a specific FIP treatment (where known: if unknown then Day 0 was the first AGP test). The normal cut-off for AGP is $500 \mu \mathrm{g} / \mathrm{ml}$. The $\mathrm{y}$ axis was cut-off at 5000 . No cat had sequential AGP tests prior to treatment, which is why it appears as if AGP was decreasing prior to treatment - in reality, it would have been increasing. AGP of the $26^{\text {th }}$ recovered cat is shown in Figure 3.

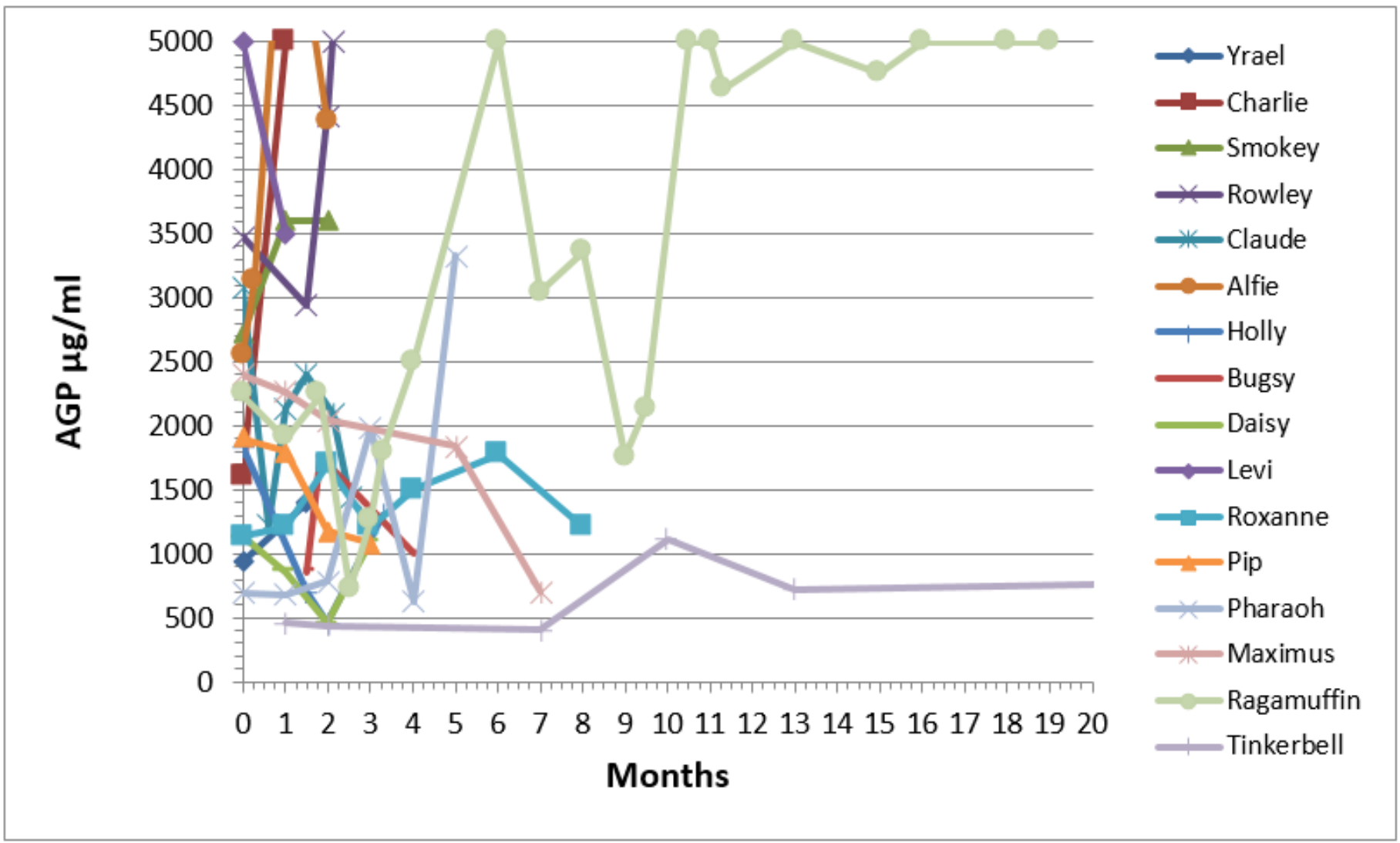

Figure 2

\section{Sequential AGP results of 16 cats who experienced remission from FIP}

This graph shows the AGP levels of cats in remission and illustrates that cats who did not fully recover from FIP retained high AGP levels: in Ragamuffin's case, very high levels. The median time for survival was 4.5 months. The AGP levels in these cats was often suppressed by prednisolone treatment. These cats are the feline equivalent of humans with long COVID. 


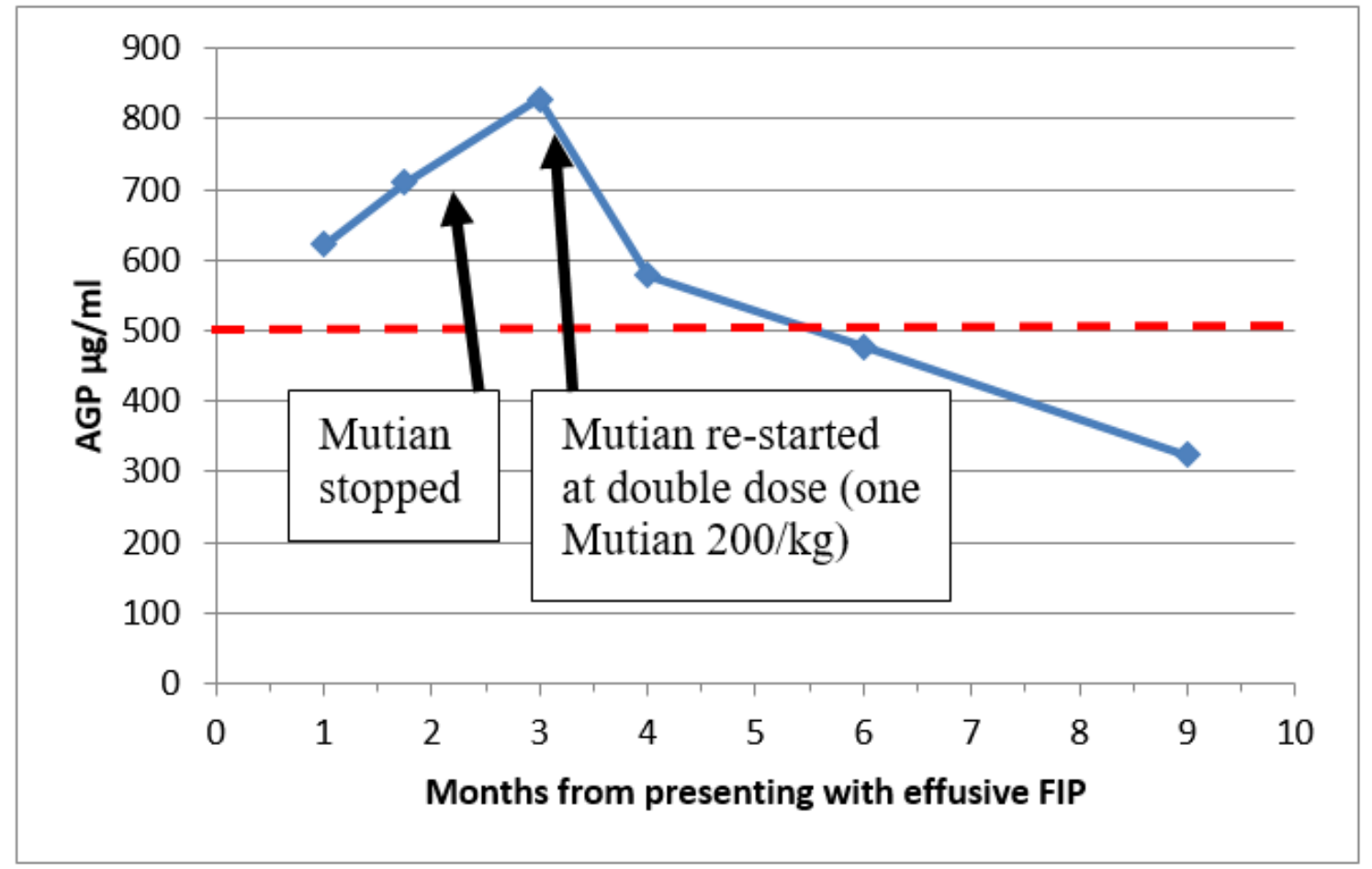

Figure 3

AGP level of Kitten 2 who relapsed.

AGP level and timeline of Kitten 2 who suffered a neurological FIP relapse. The red dashed line indicates the normal cutoff.

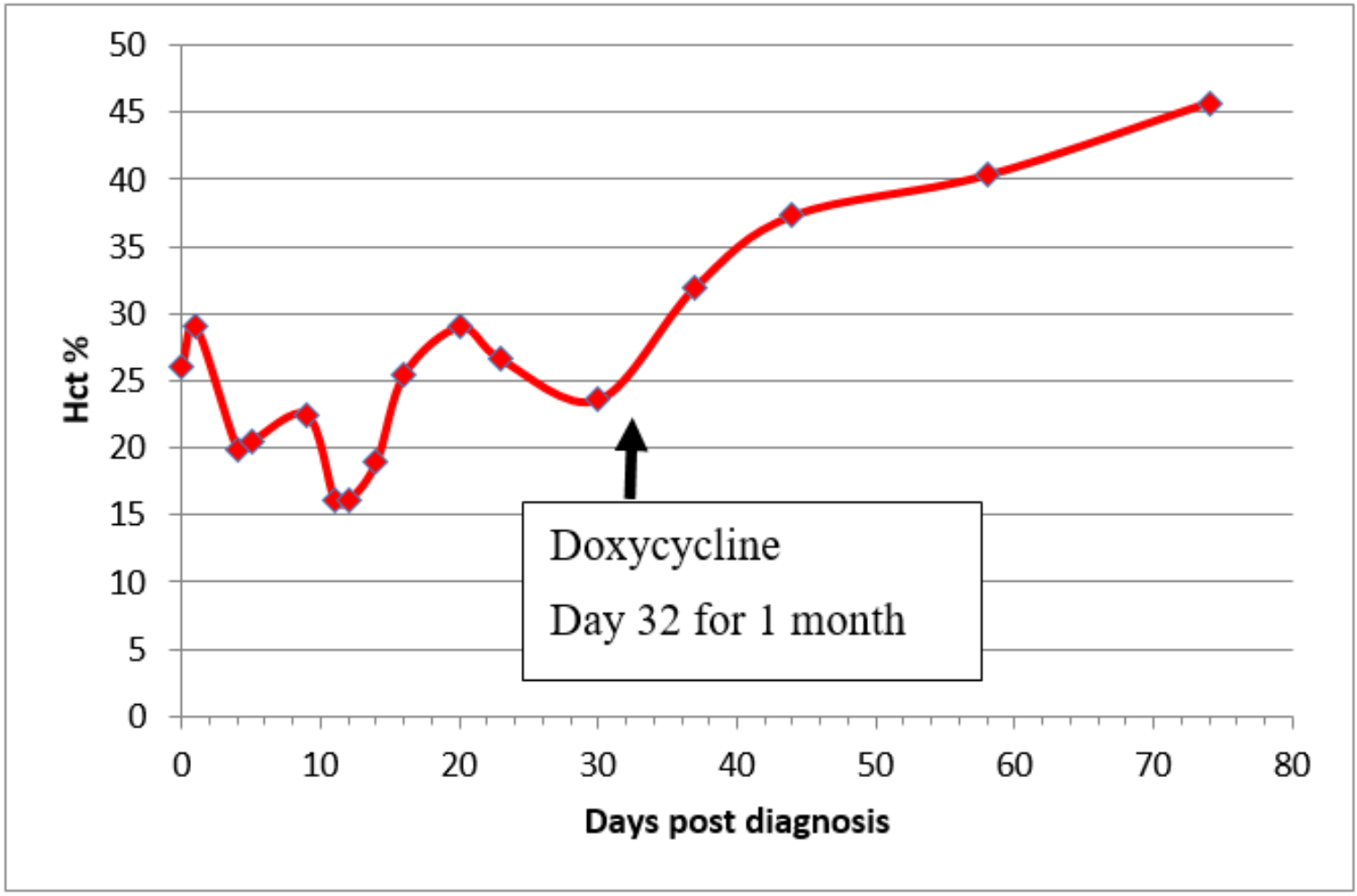

Figure 4 
Haematocrit of a cat with both FIP and haemotropic mycoplasmosis showing cyclical anaemia of parasitemia

Haematocrit (Hct) of Basil 2 showing the typical 7-10 day wave pattern of Hct following the parasitemia of haemotropic mycoplasma infection, which compounded the effect of the anaemia of FIP. Doxycycline was introduced at Day 32 and his anaemia normalised thereafter. 